Review

\title{
Mushroom Lectins: Specificity, Structure and Bioactivity Relevant to Human Disease
}

\author{
Mohamed Ali Abol Hassan ${ }^{1, \dagger}$, Razina Rouf ${ }^{1, \dagger}$, Evelin Tiralongo ${ }^{2}$, Tom W. May ${ }^{3}$ and \\ Joe Tiralongo ${ }^{1, *}$
}

1 Institute for Glycomics, Griffith University, Gold Coast Campus, Gold Coast, QLD 4222, Australia; E-Mails: ali.abolhassan@griffithuni.edu.au (M.A.A.H.); r.rouf@griffith.edu.au (R.R.)

2 School of Pharmacy and Griffith Health Institute, Griffith University, Gold Coast Campus, Gold Coast, QLD 4222, Australia; E-Mail: e.tiralongo@griffirh.edu.au

3 Royal Botanic Gardens Melbourne, South Yarra, VIC 3141, Australia; E-Mail: Tom.May@rbg.vic.gov.au

$\dagger$ These authors contributed equally to this work.

* Author to whom correspondence should be addressed; E-Mail: j.tiralongo@griffith.edu.au; Tel.: +61-7-5552-7029; Fax: +61-7-5552-8098.

Academic Editor: Patricia Berninsone

Received: 5 February 2015 / Accepted: 19 March 2015 / Published: 8 April 2015

\begin{abstract}
Lectins are non-immunoglobulin proteins that bind diverse sugar structures with a high degree of selectivity. Lectins play crucial role in various biological processes such as cellular signaling, scavenging of glycoproteins from the circulatory system, cell-cell interactions in the immune system, differentiation and protein targeting to cellular compartments, as well as in host defence mechanisms, inflammation, and cancer. Among all the sources of lectins, plants have been most extensively studied. However, more recently fungal lectins have attracted considerable attention due to their antitumor, antiproliferative and immunomodulatory activities. Given that only $10 \%$ of mushroom species are known and have been taxonomically classified, mushrooms represent an enormous unexplored source of potentially useful and novel lectins. In this review we provide an up-to-date summary on the biochemical, molecular and structural properties of mushroom lectins, as well as their versatile applications specifically focusing on mushroom lectin bioactivity.
\end{abstract}


Keywords: lectins; mushrooms; bioactivity; structure; antiproliferative activity; immunomodulatory activity; antiviral activity

\section{Introduction}

Lectins are proteins, non-immunglobulinn nature that bind diverse sugar structures with a high degree of selectivity and stereospecificity without altering the covalent structure of any recognized glycosyl ligands [1,2]. Lectins play crucial roles in various biological processes such as cellular signaling, malignancy, scavenging of glycoproteins from the circulatory system, cell-cell interactions in the immune system, differentiation and protein targeting to cellular compartments $[1,3,4]$ and, also, in host defence mechanisms, inflammation, and metastasis [5,6]. Generally lectins are able to agglutinate erythrocytes and are often referred to as hemagglutinins. However, this is an over simplification, since not all hemagglutinins are lectins. That is, not all hemagglutinins agglutinate erythrocytes through reversible binding of sugars found on the cell surface; only lectins possess this activity [7]. Lectins are widespread in distribution and have been isolated from bacteria, insects, plants seeds and roots, algae, body fluid of vertebrates, lower vertebrates, mammalian cell membranes and fungi [8]. Some viruses, including influenza, reo virus, and picorna virus, primarily use lectins to attach to host cells.

Among all the sources of lectins, plants have been most extensively studied, with notable examples including legume lectins, type 2 ribosome inactivating proteins, chitin-binding lectins, and monocot mannose-binding lectins [9-13]. These plant lectins function as the defensive system against phytopathogenic fungi and predatory animals [14,15], and they also play a role in the symbiotic relationship of plants with nitrogen fixing bacteria [16]. In animals, lectins perform a variety of biological functions, from cell adhesion to glycoprotein synthesis, as well as controlling the protein level in the blood. Some mammalian liver cell lectin receptors are believed to be responsible for the removal of certain glycoproteins from the circulatory system [17]. Animal lectins also regulate differentiation and organ formation [18], play a vital role in the migration of lymphocytes from the bloodstream into the lymphoid organs, as well as in metastasis of cancer cells [19] and drug targeting $[8,20]$.

For thousands of years mushrooms have been recognized for their medicinal properties, nutritious value and importance in spiritual ceremonies [21,22]. There have been extensive clinical trials conducted in China and Japan in order to illustrate that a number of mushrooms have medicinal and therapeutic value for the treatment/prevention of cancer, viral diseases, hypercholesterolaemia, blood platelet aggregation, and hypertension [23]. Mushrooms are essentially macrofungi that can be seen with the naked eye in contrast to microfungi, and they exhibit a distinctive fruiting body that can be hypogeous or epigeous [24]. It is estimated that there are 140,000 mushroom species that belong to the phyla Ascomycota and Basidiomycota [25]. However, only 10\% of these mushroom species are known and have been taxonomically classified, thus making them an enormous unexplored source of potentially useful substances, including lectins [26-29]. Among known mushrooms, there are around 2000 species that are edible and about 200 have traditionally been collected for food, medicine or other purposes [30]. 
In recent years mushroom lectins have attracted considerable attention due to their antitumor, antiproliferative and immunomodulatory activities [19,31-38]. In this review, we provide an update, which builds on a series of reviews on mushroom lectins [19,36,38-41], that gives an up-to-date summary of the biochemical, molecular and structural properties of mushroom lectins, as well as their versatile applications specifically focusing on mushroom lectin bioactivity.

\section{Mushroom Lectins}

Mushrooms express high levels of lectins as storage proteins, that are thought to have a potential role in defence, similar to plant lectins [36,42]. In addition, a significant role for lectins is emerging in relation to symbiotic associations between fungi and other organisms, such as in mycorrhizas and lichens, and in cell interactions with respect to flocculation, mycelial aggregation and mating $[43,44]$. Mushroom lectins isolated from different species vary in molecular masses, subunit number and carbohydrate specificity (Table 1) [36], but lectins with very different biochemical properties have also been isolated from a single species [45,46]. Moreover, lectins have been purified from different parts of the mushroom, including caps, stalks, and mycelia, and the expression levels may vary depending on fruit-body age [47] and season [40]. For example, the quantity of Laccaria laccata lectin is higher in adult mushrooms whereas the expression of lectin from Amanita muscaria, Tricholomopsis rutilans and Lactarius rufus is higher in young mushrooms. Similarly, we have reported on mushrooms collected in Australia, in which the expression of lectin varies with respect to environmental influences, such as season, location, and year, as well as depending on macroscopic properties, such as age and mycelia growth [7].

Table 1. Mushroom lectins and their specificity.

\begin{tabular}{|c|c|c|}
\hline $\begin{array}{l}\text { Source of Lectin (Current Species } \\
\text { Names Given in Parentheses) }\end{array}$ & Specificity of Sugars/Glycoproteins * & Ref. \\
\hline Agaricus arvensis & Inulin & {$[48]$} \\
\hline Agaricus bisporus & GalNAc, Gal $\beta 1,3$ GalNAc (T antigen), sialyl-Gal $\beta$ (ABL) & [49-52] \\
\hline Agaricus bitorquis & $\mathrm{Lac}$ & {$[7]$} \\
\hline $\begin{array}{l}\text { Agaricus blazei } \\
\text { (Agaricus subrufescens) }\end{array}$ & $\begin{array}{l}\text { Methyl } N \text {-acetyl- } \alpha \text {-o-galactosaminide, GalNAc, BSM, } \\
\text { asialo-BSM, fetuin, asialofetuin }\end{array}$ & {$[53]$} \\
\hline Agaricus campestris & GalNAc, Gal, Suc & {$[54,55]$} \\
\hline Agaricus pilatianus & Lac, GlcNAc, Glc, Rham & {$[47]$} \\
\hline $\begin{array}{l}\text { Agrocybe aegerita } \\
\text { (Cyclocybe aegerita) }\end{array}$ & $\begin{array}{l}\text { Lac, BSM, Glycophorin A, k-Casein, } \beta \text {-galactosides, Gal (AAL galectin), } \\
\text { terminal non-reducing GlcNAc (AAL2) }\end{array}$ & [56-59] \\
\hline $\begin{array}{l}\text { Agrocybe cylindracea } \\
\text { (Cyclocybe cylindracea) }\end{array}$ & Trisaccharides containing Neu $5 \mathrm{Ac} \alpha 2,3 \mathrm{Gal}$, Lac, sialic acid, inulin (ACG) & [60-63] \\
\hline Aleuria aurantia & L-Fuc, fucosyl oligosaccharides (AAL) & {$[64,65]$} \\
\hline Amanita muscaria & $O$-type glycans & {$[66]$} \\
\hline Amanita ovoidea & Gal, GalNAc, Rham & {$[47]$} \\
\hline Amantia pantherina & $\begin{array}{l}\text { GIcNAc } \beta 1,4 \mathrm{Man} \beta \mathrm{pNP}, \text { Gal } \beta 1,4 \mathrm{GlcNAc} \beta 1,4 \mathrm{GIcNAc}, \\
\text { Gal } \beta 1,4 \mathrm{GIcNAc} \beta 1,4 \mathrm{GlcNAc}, \mathrm{BSM}, \text { asialo-BSM }\end{array}$ & {$[67]$} \\
\hline Amanita phalloides & Ovomucin, human glycophorin A (A. phalloides lectin) & {$[66]$} \\
\hline
\end{tabular}


Table 1. Cont.

\begin{tabular}{|c|c|c|}
\hline $\begin{array}{l}\text { Source of Lectin (Current Species } \\
\text { Names Given in Parentheses) }\end{array}$ & Specificity of Sugars/Glycoproteins * & Ref. \\
\hline Amanita virosa & $\begin{array}{l}\text { Blood group specific substance } \mathrm{B}, \mathrm{A} \text { and } \mathrm{H} \text {, bovine thyreoglobulin, } \\
\text { ovomucoid, asialo-ovomucoid transferrin, Ovine submaxillary mucin, } \\
\text { 4-nitrophenyl- } \alpha \text {-D-mannopyranoside, 4-nitrophenyl- } \beta \text {-D-glucopyranoside, } \\
\text { 4-nitrophenyl- } \beta \text {-D-galactopyranoside }\end{array}$ & {$[68]$} \\
\hline Amanita muscaria & $O$-type glycans & {$[66]$} \\
\hline Armillaria luteovirens & Inulin (ALL) & [69] \\
\hline $\begin{array}{l}\text { Auricularia polytricha } \\
\text { (Auricularia cornea) }\end{array}$ & $\begin{array}{l}\text { Raf, Gal, ovomucoid and } \beta \text {-anomers of galactoside } \\
\text { (Lac, } p \text {-nitrophenyl } \beta \text {-D-galactoside) }\end{array}$ & {$[70]$} \\
\hline $\begin{array}{l}\text { Boletopsis leucomelaena } \\
\text { [as "leucomelas"] } \#\end{array}$ & $\begin{array}{l}\text { GlcNAc } \beta 1,2 \text { Man } \alpha 1,3(\text { GlcNAc } \beta 1,2 \text { Man } \alpha 1,6) \text { Man } \beta 1,4 \text { GlcNAc } \beta 1, \\
\text { 4GlcNAc, GlcNAc (BLL) }\end{array}$ & {$[71]$} \\
\hline Boletus edulis & $\mathrm{D}(+)-\mathrm{Mel}, \mathrm{D}-\mathrm{Xyl}(\mathrm{BEL})$ & {$[72]$} \\
\hline Boletus satanas & D-Gal & {$[73]$} \\
\hline $\begin{array}{l}\text { Boletus subtomentosus } \\
\text { (Xerocomus subtomentosus) }\end{array}$ & D-Lac & {$[74]$} \\
\hline Boletus venenatus & $\begin{array}{l}\text { Asialofetuin, Gal } \beta 1,4 \mathrm{GlcNAc} \beta 1,4 \mathrm{Man} \beta 1,4 \mathrm{GlcNAc} \beta 1,4 \mathrm{GlcNAc} \\
\text { residues in } N \text {-linked sugar chains }\end{array}$ & {$[75]$} \\
\hline Chlorophyllum brunneum & Neu5Ac & [7] \\
\hline Chlorophyllum molybdites & Neu5Gc, GalNAc, asialo-BSM, PSM & {$[76]$} \\
\hline Ciborinia camelliae & GalNAc & {$[77]$} \\
\hline $\begin{array}{l}\text { Clavaria purpurea } \\
\text { (Alloclavaria purpurea) }\end{array}$ & Asialo-BSM, $\alpha$-Gal, Gal $\alpha 1,3 \mathrm{Gal}, \mathrm{Raf}$ & {$[78]$} \\
\hline Clitocybe geophyla $\wedge^{\wedge}$ & GalNAc, Lac, Glc & {$[47]$} \\
\hline Clitocybe nebularis & $\begin{array}{l}\text { Asialo-fetuin, Lac, GalNAc, } \\
\text { Gal, } N, N \text {-diacetyllactosediamine (GalNAc } \beta 1,4 \mathrm{GlcNAc} \text {, LacdiNAc) (CNL) }\end{array}$ & [79-81] \\
\hline $\begin{array}{l}\text { Coprinus atramentarius } \\
\text { (Coprinopsis atramentaria) }\end{array}$ & D-Lac & {$[74]$} \\
\hline Coprinus comatus & GlcNAc, Lac, Gal, Ara, Rib, Xyl & {$[7,47]$} \\
\hline $\begin{array}{l}\text { Coprinus cinereus } \\
\text { (Coprinopsis cinerea) }\end{array}$ & $\begin{array}{l}\beta \text {-Gal (CCL2), GlcNAc } \beta 1,4 \text { (Fuc } \alpha 1,3) \text { GlcNAc (CGL2), } \\
\text { GalNAc } \beta 1,4 \text { GlcNAc (CGL3) }\end{array}$ & [82-84] \\
\hline $\begin{array}{l}\text { Coprinus micaceus } \\
\text { (Coprinellus micaceus) }\end{array}$ & Lac, Gal, GalNAc & {$[47]$} \\
\hline Cordyceps militaris & Sialoglycoprotein, Neu5Ac (CML) & [85] \\
\hline Cortinarius sp. TWM 1710 & Gal & [7] \\
\hline Flammulina velutipes & $\begin{array}{l}\beta \text {-D-Gal, fetuin, human transferrin, human glycophorin, } \\
\text { lactoferrin (F. velutipes lectin) }\end{array}$ & {$[86,87]$} \\
\hline Fomes fomentarius & GalNAc, $\alpha$-D-Gal, Raf & {$[80]$} \\
\hline Ganoderma capense & D-Gal, $\mathrm{D}(+)$-Galactosamine (G. capense lectin) & {$[88]$} \\
\hline Ganoderma lucidum & Asialo-triantennary $N$-glycan, $N$-and $O$-linked glycans. & {$[89,90]$} \\
\hline Grifola frondosa & $\begin{array}{l}\text { Terminal GalNAc residues, porcine stomach mucin, linear D-Rham, } \\
\text { PSM (GFL) }\end{array}$ & {$[76,91,92]$} \\
\hline $\begin{array}{l}\text { Hericium erinaceus } \\
\text { [as “erinaceum"] }{ }^{\#}\end{array}$ & Neu5Gc, Neu5Ac, inulin (HEA) & {$[62,93,94]$} \\
\hline
\end{tabular}


Table 1. Cont.

\begin{tabular}{|c|c|c|}
\hline $\begin{array}{l}\text { Source of Lectin (Current Species } \\
\text { Names Given in Parentheses) }\end{array}$ & Specificity of Sugars/Glycoproteins * & Ref. \\
\hline Hygrophorus hypothejus & $\begin{array}{l}\text { Lac, D-Gal, D-GalNAc, Gal } \beta 1,4 \mathrm{GlcNAc}, o \text {-nitrophenyl } \alpha \text {-D-GalNAc, } \\
p \text {-nitrophenyl- } \beta \text {-D-GalNAc, asialo-BSM }\end{array}$ & {$[95,96]$} \\
\hline Hygrophorus russula & $\begin{array}{l}\alpha 1,6 \text {-mannobiose, Isomaltose (Glc } \alpha 1,6 \mathrm{Glc}) \text {, isomaltotriose, } \\
\text { isomaltotetraose, isomaltopentaose, isomaltohexaose, methyl } \alpha \text {-mannoside, } \\
\alpha 1,3 \text {-mannobiose, methyl } \beta \text {-mannoside, } \alpha 1,2 \text {-mannobiose, } \\
\alpha 1,4 \text {-mannobiose, methyl } \alpha \text {-glucoside, Man, lacturose (HRL) }\end{array}$ & [97] \\
\hline $\begin{array}{l}\text { Inocybe fastigiata } \\
\text { (Inocybe rimosa) }\end{array}$ & GalNAc & {$[98]$} \\
\hline Inocybe umbrinella & Raf, D-Mel, $\alpha$-Lac, D-Gal (I. umbrinella lectin) & [99] \\
\hline Ischnoderma resinosum & Methyl- $\beta$-galactoside, Fuc, L-Ara & {$[53]$} \\
\hline Kuehneromyces mutabilis & Asialo-PSM, asialofetuin, fetuin, $\alpha 1$-acid glycoprotein, Ovomucoid & {$[100]$} \\
\hline Laccaria amethystina & $\begin{array}{l}\text { L-Fuc (LAF), D-Lac and GalNAc, BSM, asialo-BSM,PSM, asialo-PSM, } \\
\text { human glycophorin A (LAL) }\end{array}$ & {$[101,102]$} \\
\hline Laccaria laccata & L-Fuc & {$[103]$} \\
\hline Lactarius deliciosus & Gal $\beta 1,3$ GalNAc & {$[104]$} \\
\hline Lactarius deterrimus & Gal $\beta 1,3$ GalNAc & {$[105]$} \\
\hline Lactarius flavidulus & $\begin{array}{l}\text { D-Mel, D-Fru, L(+)-Rham, Sor, D-Gal, D(+)-Man, Lac, D(+)-Xyl, L(+)-Ara, } \\
\text { D-Glu, Raf, Inulin, } p \text {-nitrophenyl- } \alpha, \mathrm{D}-\text { glucopyranoside, } \\
p \text {-nitrophenyl- } \beta, \mathrm{D}-\text { glucopyranoside, inositol (LFL) }\end{array}$ & {$[106]$} \\
\hline Lactarius lignyotus & Asialofetuin, asialo-PSM and other desialylated glycoproteins & {$[107]$} \\
\hline Lactarius pergamenus & $\begin{array}{l}\text { GalNAc, 4-nitrophenyl- } \beta \text {-D-galactopyranoside, } \\
\alpha \text {-phenyl } N \text {-acetyl-D-glucosaminopyranoside, Bovine thyroglobulin, } \\
\text { human transferrin, Orosomucoid ( } \alpha \text {-glycoprotein), } \\
\text { sheep submaxillary mucin, BSM, asialo-BSM, fetuin }\end{array}$ & [108] \\
\hline Lactarius rufus & $\begin{array}{l}\alpha \text {-phenyl } N \text {-acetyl-D-glucosaminopyranoside, } \\
\text { 4-nitrophenyl- } \beta \text {-D-glucosamine, asialo-BSM, human and bovine } \\
\text { thyroglobulin, group specific substances from human erythrocytes }\end{array}$ & [109] \\
\hline Lactarius salmonicolor & Gal $\beta 1,3$ GalNAc & {$[110]$} \\
\hline $\begin{array}{l}\text { Laetiporus sulphureus } \\
\text { [as "sulfureus"] }{ }^{\#}\end{array}$ & $\begin{array}{l}\text { LacNAc, L-Rham, salicine, asialo-BSM,BSM, asialofetuin, } \\
\text { lacto- } N \text {-neotetraose (Gal } \beta 1,4 \mathrm{GlcNAc} \beta 1,3 \mathrm{Gal} \beta 1,4 \mathrm{Glc})(\mathrm{LSL})\end{array}$ & [111-113] \\
\hline $\begin{array}{l}\text { Lentinus edodes } \\
\text { (Lentinula edodes) }\end{array}$ & GlcNAc, GalNAc, Man, D-Mel, Gal & [114-117] \\
\hline Lentinus squarrosulus & Raf, D-Suc, Rib & {$[118]$} \\
\hline $\begin{array}{l}\text { Lepiota leucothites } \\
\text { (Leucoagaricus leucothites) }\end{array}$ & Glc, GlcNAc, Man & [47] \\
\hline $\begin{array}{l}\text { Lepiota rhacodes } \\
\text { (Macrolepiota rachodes) }\end{array}$ & Lac, Arab, MethGLc, GalNAc & {$[47]$} \\
\hline Lepista nuda & Gal,Fuc, Suc, Arab & {$[47]$} \\
\hline Lyophyllum decastes & Galabiose-Gal $\alpha 1,4 \mathrm{Gal}$, non-reducing $\alpha$-Gal & {$[119]$} \\
\hline Macrolepiota procera & Terminal $N$-acetyl-lactosamine, $\beta$-galactosides (MPL) & {$[120]$} \\
\hline
\end{tabular}


Table 1. Cont.

\begin{tabular}{|c|c|c|}
\hline $\begin{array}{l}\text { Source of Lectin (Current Species } \\
\text { Names Given in Parentheses) }\end{array}$ & Specificity of Sugars/Glycoproteins * & Ref. \\
\hline Marasmius oreades & $\begin{array}{l}\text { Gal } \alpha 1,3 \mathrm{Gal} \beta 1,4 \mathrm{GlcNAc} \text {, blood group Btrisaccharide (Gal } \alpha 1,3 \mathrm{Gal} 2,1 \alpha \mathrm{Fuc} \text { ), } \\
\text { Man, thyroglobulin, asialofetuin, complex type } N \text {-glycans (MOA) }\end{array}$ & {$[121-125]$} \\
\hline Melanoleuca brevipes & Gal, Rham, Lac & [47] \\
\hline Melastiza chateri & L-Fuc & {$[126]$} \\
\hline Mycoleptodonoides aitchisonii & Asialo-BSM, BSM & [127] \\
\hline Omphalotus nidiformis & Lac, Gal, Ara, Rib & [7] \\
\hline $\begin{array}{l}\text { Oudemansiella platyphylla } \\
\text { (Megacollybia platyphylla) }\end{array}$ & $\beta$-GalNAc, terminal GlcNAc & {$[47,76,128]$} \\
\hline Panus conchatus & $\mathrm{D}-\mathrm{Gal}$ & {$[40,129]$} \\
\hline Paxillus involutus & Asialo-PSM, asialofetuin, fetuin, $\alpha 1$-acid glycoprotein (P. involutus lectin) & {$[103]$} \\
\hline Paecilomyes japonica $^{\wedge}$ & Sialic acid and sialoglycoprotein (PJA) & {$[130]$} \\
\hline $\begin{array}{l}\text { Peziza silvestris [as "sylvestris"] \# } \\
\text { (Peziza arvernensis) }\end{array}$ & L-Ara ( $P$. silvestris lectin) & [131] \\
\hline Phaeolepiota aurea & GalNAc (PAL1 and PAL2) & [132] \\
\hline Phallus impudicus & Fetuin & {$[76]$} \\
\hline Phlebopus marginatus & Lac, Gal & {$[7]$} \\
\hline Pholiota adiposa & Inulin (PAL) & {$[133]$} \\
\hline Pholiota aurivella & Asialofetuin & [134] \\
\hline Pholiota squarrosa & $\alpha 1,6$-fucosylated $N$-glycans & {$[135]$} \\
\hline Pleurocybella porrigens & GalNAc, asialo-BSM, $O$-linked glycans & {$[136]$} \\
\hline Pleurotus citrinopileatus & $\begin{array}{l}\text { Mal, } o \text {-nitrophenyl- } \beta \text {-D-galactopyranoside, } \\
o / p \text {-nitrophenyl- } \beta \text {-D-glucuronide, inulin (P. citrinopileatus lectin) }\end{array}$ & [137] \\
\hline Pleurotus cornucopiae & Asialo-mucin & {$[138]$} \\
\hline Pleurotus eous & Methyl- $\alpha$-D-galactoside, galactosamine, mannosamine, asialofetuin (PEL) & {$[139]$} \\
\hline Pleurotus ostreatus & $\begin{array}{l}\text { Me- } \alpha \text {-GalNAc and 2'-fucosyllactose (Fuc } \alpha 1,2 \mathrm{Gal} \beta 1,4 \mathrm{Glc}) \text {, D-Mel, D-Gal, } \\
\text { Raf, NeuNAc, Inulin, Lac, Galactosyl and } N \text {-Acetyl galactosaminyl groups, } \\
\text { BSM, asialo-BSM (POL) }\end{array}$ & {$[32,140-142]$} \\
\hline $\begin{array}{l}\text { Pleurotus serotinus } \\
\text { (Sarcomyxa serotina) }\end{array}$ & GalNAc & [129] \\
\hline Pleurotus spodoleucus & Lac & {$[140]$} \\
\hline Pleurotus tuber-regium & GlcNAc & [143] \\
\hline $\begin{array}{l}\text { Polyporus adustus [as "adusta"] " } \\
\text { (Bjerkandera adusta) }\end{array}$ & $\begin{array}{l}\text { D-Mel, D-Fru, D-Ara, D-Glu, D-Raf, } p \text {-nitro- } \alpha \text {-D-glucopyranoside } \\
\text { (P. adustus lectin) }\end{array}$ & {$[144]$} \\
\hline Polyporus squamosus & $\begin{array}{l}\text { Neu5Ac } 22,6 \mathrm{Gal} 1,4 \mathrm{Glc} / \mathrm{GlcNAc} \text { (6'-sialylated type II chain) } \\
\text { of } N \text {-glycans (PSL) }\end{array}$ & {$[145-148]$} \\
\hline Psathyrella asperospora & GlcNAc (PAL) & {$[7]$} \\
\hline Psathyrella velutina & GlcNAc, Neu5Aca2,3Galß1,4GlcNAc, Heparin and Pectin (PVL) & {$[62,149-152]$} \\
\hline Psilocybe barrerae & D-Gal, Glycophorin, BSM, asialo-BSM, human serum and milk transferrin & {$[153]$} \\
\hline Russula delica & Inulin, $o$-nitrophenyl- $\beta$-D-galactopyranoside ( $R$. delica lectin) & [154] \\
\hline Russula lepida & Inulin, $o$-nitrophenyl- $\beta$-D-galactopyranoside & [155] \\
\hline Russula nigricans & Asialofetuin, asialo-PSM, fetuin, ovomucoid, $\alpha 1$-acid glycoprotein & {$[103]$} \\
\hline
\end{tabular}


Table 1. Cont.

\begin{tabular}{|c|c|c|}
\hline $\begin{array}{l}\text { Source of Lectin (Current Species } \\
\text { Names Given in Parentheses) }\end{array}$ & Specificity of Sugars/Glycoproteins * & Ref. \\
\hline Schizophyllum commune & $\begin{array}{l}\text { GalNAc (S. commune lectin, species from Thailand), } \\
\text { Lac (S. commune lectin, species from China) }\end{array}$ & {$[156,157]$} \\
\hline Stereum hirsutum & L-Xyl & [158] \\
\hline Trametes versicolor & Gal & {$[47]$} \\
\hline $\begin{array}{l}\text { Tricholoma fracticum } \\
\text { [as "fractum"] } \#\end{array}$ & Lac, Gal, GalNAc & {$[47]$} \\
\hline $\begin{array}{l}\text { Tricholoma mongolicum } \\
\text { (Leucocalocybe mongolica) }\end{array}$ & Lac (TML1), GalNAc and Gal (TML2) & {$[45]$} \\
\hline Volvariella volvacea & Thyroglobulin (VVL) & [31] \\
\hline Xerocomus chrysenteron & $\begin{array}{l}\text { Asialofetuin, asialo-PSM and other desialyzed glycoproteins Sychrova, } \\
\text { GalNAc, Gal,TF antigen (XCL) }\end{array}$ & {$[107,159,160]$} \\
\hline $\begin{array}{l}\text { Xerocomus spadiceus } \\
\text { (Xerocomus ferrugineus) }\end{array}$ & Inulin ( $X$. spadiceus lectin) & {$[161]$} \\
\hline Xylaria hypoxylon & Inulin, Xyl (X. hypoxylon lectin) & {$[162]$} \\
\hline \multicolumn{3}{|c|}{$\begin{array}{l}\text { * Ara, arabinose; BSM, Bovine submaxillary mucin; Fru, fructose; Gal, galactose; Glu, glucose; GalNAc, } \\
N \text {-acetyl-D-galactosamine; GlcNAc, } N \text {-acetyl-D-glucosamine; Lac, lactose; Man, mannose; Mel, Melibiose; } \\
\text { Neu5Gc, } N \text {-glycolyl-neuraminic acid; Neu5Ac, } N \text {-acetyl-neuraminic acid; PSM, Procine submaxillary mucin } \\
\text { Raf, raffinose; Rham, rhamnose; Rib, ribose; Sor, sorbitol, Suc, sucrose; Xyl, xylose; "The species name was } \\
\text { incorrectly spelt in the original publication (in square brackets), the correctly spelt name has now been } \\
\text { provided; ^ Names could not be matched to the global fungi name databases. "Clitocybe geophyla" as } \\
\text { reported by Mikiashvili et al. (2006) [47] could be "Clitocybe geotropa" or "Inocybe geophylla". } \\
\text { "Paecilomyces japonica" as reported by Park et al. (2004) [127] could be Isaria japonica Yasuda, in which } \\
\text { case the current name is Isaria tenuipes following Luangsa-ard et al. (2005) [163]; "These abbreviated lectin } \\
\text { names are provided for those mushroom species that are mentioned in Sections } 4 \text { and } 5 \text { and also for } \\
\text { mushroom species that have two or more lectins. }\end{array}$} \\
\hline
\end{tabular}

The first mushroom lectin "phallin" was described in Amanita phalloides, a hemolytic agent [164]. Approximately 105 lectins have been identified in diverse mushroom species. Table 1 gives a complete list of lectins, thus far identified in mushroom, and their carbohydrate and/or glycoprotein specificity. The species names given in Table 1 have been updated (in parentheses) where appropriate predominantly using Species Fungorum (http://www.speciesfungorum.org/), and the spelling has also been corrected for some species names. Up-to-date nomenclature, especially in terms of correct generic placement, allows comparisons with phylogenetically related taxa and also facilitates targeted collecting of closely related species for the identification and isolation of novel lectins. Even though we have updated and corrected species names in Table 1, to avoid confusion we have used the originally species names (as published) from which the lectin(s) were identified in this review.

The largest number of lectins has been identified from Lactarius followed by Pleurotus, Agaricus, Amanita and Boletus. Interestingly, there are a number of mushroom species from which more than one lectin has been isolated, for example Coprinus cinereus [82-84], Agrocybe aegerita [56-59], Agrocybe cylindracea [60,61], Laccaria amethystina [101] and Schizophyllum commune [156,157]. Mushrooms species where multiple lectins have been identified, and those that are further discussed 
and listed in Tables 2-4, the published lectin abbreviations have been given in Table 1 . As illustrated in Table 1, mushroom lectins have been identified with varying sugar specificities, from lectins that only bind the polysaccharide inulin (e.g., lectins from Agaricus arvensis [48], Pholiota adiposa [133], Xerocomus spadiceus [161]) to lectins that bind lactose (Agaricus bitorquis, Boletus subtomentosus, Coprinus atramentarius, Pleurotus spodoleucus [7,74,140]), galactose (Boletus sataaus and Panus conchatus [40,73,129]), N-acetylgalactosamine (Ciborinia camelliae, Inocybe fastigiata, Lactarius vellereus, Pleurotus serotinus [77,98,129]), and sialic acid (Hericium erinaceus, Polyporus squamosus, Psathyrella velutina, Paecilomyes japonica, and Agrocybe cylindracea [62]). 
Table 2. Common structural characteristics and strategies used to purify mushroom lectins for which crystal structure data exists.

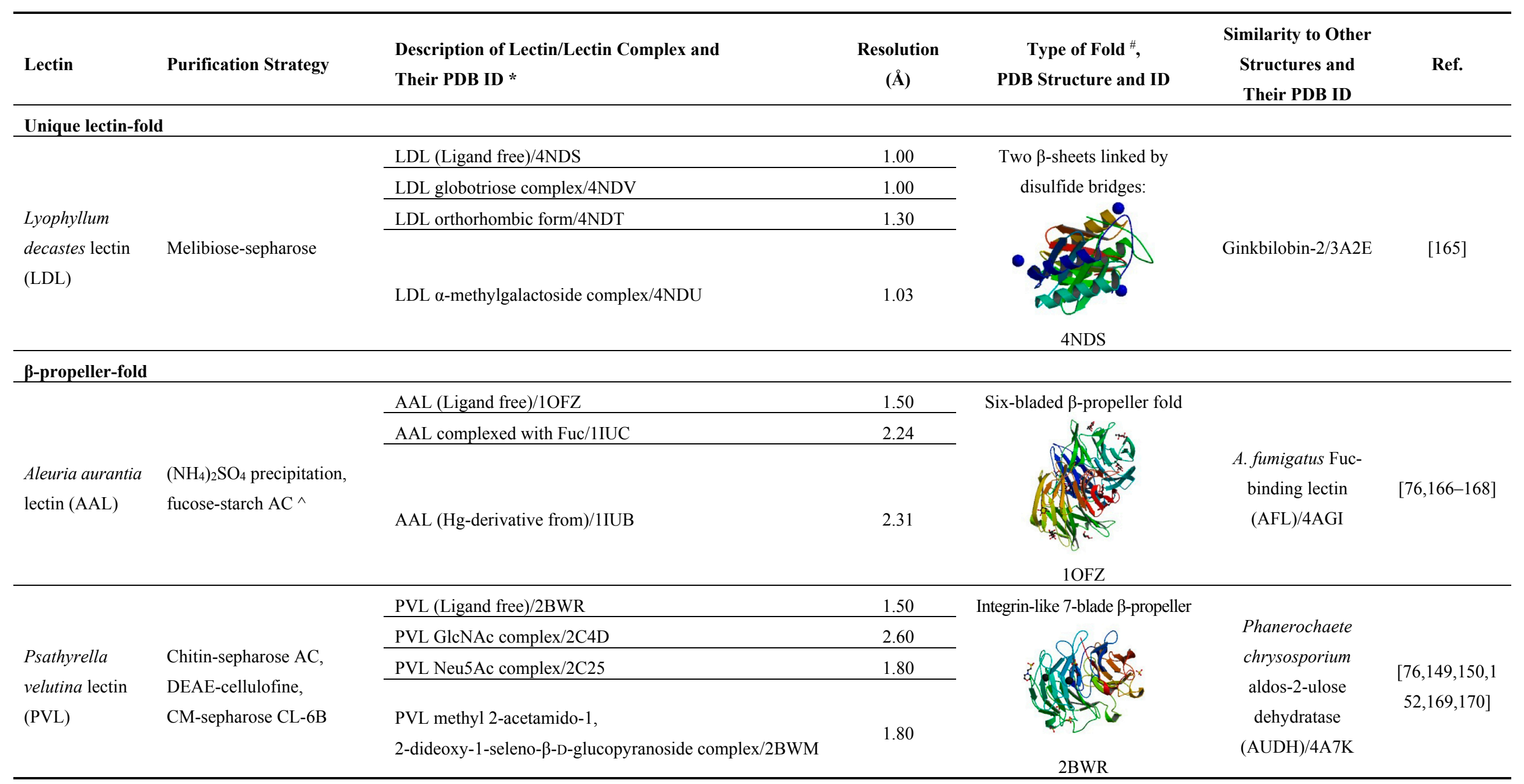


Table 2. Cont

\begin{tabular}{|c|c|c|c|c|c|c|}
\hline Lectin & Purification Strategy & $\begin{array}{l}\text { Description of Lectin/Lectin Complex and } \\
\text { Their PDB ID * }\end{array}$ & $\begin{array}{l}\text { Resolution } \\
\text { (Å) }\end{array}$ & $\begin{array}{l}\text { Type of Fold } \\
\text { PDB Structure and ID }\end{array}$ & $\begin{array}{c}\text { Similarity to Other } \\
\text { Structures and } \\
\text { Their PDB ID } \\
\end{array}$ & Ref. \\
\hline \multicolumn{7}{|c|}{ Galectin-like fold } \\
\hline \multirow{16}{*}{$\begin{array}{l}\text { Agrocybe } \\
\text { aegerita } \\
\text { lectin/galectin } \\
\text { (AAL-galectin) }\end{array}$} & \multirow{16}{*}{$\begin{array}{l}\left(\mathrm{NH}_{4}\right)_{2} \mathrm{SO}_{4} \text { precipitation, } \\
\text { DEAE-sepharose FF, } \\
\text { Sephacryl S-200 HR, } \\
\text { GF-250 HPLC }\end{array}$} & AAL-galectin (Ligand free)/2ZGK & 3.00 & \multirow{16}{*}{ s): } & \multirow{16}{*}{$\begin{array}{c}\text { ACG complexed } \\
\text { with blood type A } \\
\text { antigen } \\
\text { tetraose/3WG3 }\end{array}$} & \multirow{16}{*}[171,172]{} \\
\hline & & $\begin{array}{l}\text { Recombinant AAL-galectin (rAAL-galectin) } \\
\text { (Ligand free)/2ZGL }\end{array}$ & 1.90 & & & \\
\hline & & rAAL-galectin Lac complex/2ZGM & 1.90 & & & \\
\hline & & rAAL-galectin Gal complex/2ZGN & 2.50 & & & \\
\hline & & AAL-galectin mutant H59Q Lac complex/2ZGO & 2.00 & & & \\
\hline & & AAL-galectin mutant I25G/2ZGP & 2.70 & & & \\
\hline & & AAL-galectin mutant L33A/2ZGQ & 1.90 & & & \\
\hline & & AAL-galectin mutant L33A/2ZGR & 1.90 & & & \\
\hline & & AAL-galectin mutant L47A/2ZGS & 1.90 & & & \\
\hline & & AAL-galectin mutant F93G/2ZGT & 2.80 & & & \\
\hline & & AAL-galectin mutant I144G/2ZGU & 2.40 & & & \\
\hline & & AAL-galectin TF antigen complex/3AFK & 1.95 & & & \\
\hline & & AAL-galectin $p$-nitrophenyl TF disaccharide complex/3M3C & 2.00 & & & \\
\hline & & $\begin{array}{l}\text { AAL-galectin mutant E66A p-nitrophenyl TF } \\
\text { disaccharide complex/3M3E }\end{array}$ & 2.10 & & & \\
\hline & & $\begin{array}{l}\text { AAL-galectin mutant R85A p-nitrophenyl TF } \\
\text { disaccharide complex/3M } 3 \mathrm{O}\end{array}$ & 2.10 & & & \\
\hline & & $\begin{array}{l}\text { AAL-galectin ganglosides complex GM1 } \\
\text { pentasaccharide/3M3Q }\end{array}$ & 2.10 & & & \\
\hline
\end{tabular}


Table 2. Cont.

\begin{tabular}{|c|c|c|c|c|c|c|}
\hline Lectin & Purification Strategy & $\begin{array}{l}\text { Description of Lectin/Lectin Complex and } \\
\text { Their PDB ID * }\end{array}$ & $\begin{array}{l}\text { Resolution } \\
\quad(\AA)\end{array}$ & $\begin{array}{c}\text { Type of Fold ", } \\
\text { PDB Structure and ID }\end{array}$ & $\begin{array}{c}\text { Similarity to Other } \\
\text { Structures and } \\
\text { Their PDB ID } \\
\end{array}$ & Ref. \\
\hline \multicolumn{7}{|c|}{ Galectin-like fold } \\
\hline \multirow{7}{*}{$\begin{array}{l}\text { Agrocybe } \\
\text { cylindracea } \\
\text { galectin/lectin } \\
\text { (ACG) }\end{array}$} & \multirow{7}{*}{$\begin{array}{l}\left(\mathrm{NH}_{4}\right)_{2} \mathrm{SO}_{4} \text { precipitation, } \\
\text { DEAE-cellulofine A-200, } \\
\text { DEAE-Toyopearl } 650 \mathrm{M} \text {, } \\
\text { and Toyopearl HW }\end{array}$} & ACG (Ligand free)/1WW7 & 1.90 & \multirow{7}{*}{$1 \mathrm{WW} 7$} & \multirow{7}{*}{$\begin{array}{c}\text { Recombinant } \\
\text { (rAAL-galectin)/ } \\
\text { 2ZGL }\end{array}$} & \multirow{7}{*}[60,173,174]{} \\
\hline & & ACG Lac complex/1WW6 & 2.20 & & & \\
\hline & & ACG 3'-sulfonyl Lac complex/1WW5 & 2.20 & & & \\
\hline & & ACG $\alpha 2,3$-sialyllactose complex/1WW4 & 2.30 & & & \\
\hline & & ACG mutant (N46A) blood type A & 1.60 & & & \\
\hline & & antigen tetraose complex/3WG4 & 1.60 & & & \\
\hline & & ACG blood type A antigen tetraose complex/3WG3 & 1.35 & & & \\
\hline \multirow{7}{*}{$\begin{array}{l}\text { Coprinus } \\
\text { cinereus lectin } \\
\text { (CGL2) }\end{array}$} & \multirow{7}{*}{ Lactosyl-sepharose AC } & CGL2 (Ligand free)/1UL9 & 2.22 & \multirow{7}{*}{ 1UL9 } & \multirow{7}{*}{$\begin{array}{l}\text { CGL3 chitotetraose } \\
\text { complex/2R0H }\end{array}$} & \multirow{7}{*}[82,175,176]{} \\
\hline & & CGL2 Lac complex/1ULC & 2.60 & & & \\
\hline & & CGL2 xeno linear trisaccharide complex/1ULE & 2.15 & & & \\
\hline & & CGL2 blood group A tetrasaccharide complex/1ULF & 2.36 & & & \\
\hline & & CGL2 TF antigen complex/1ULG & 2.20 & & & \\
\hline & & CGL2 blood H Type II complex/1ULD & 2.20 & & & \\
\hline & & CGL2 C. elegans $N$-glycan complex/2WKK & 2.10 & & & \\
\hline \multirow{3}{*}{$\begin{array}{l}\text { Coprinopsis } \\
\text { cinerea lectin } \\
\text { (CGL3) }\end{array}$} & \multirow{3}{*}{ Lactosyl-sepharose AC } & CGL3 (Ligand free)/2R0F & 2.00 & & \multirow{3}{*}{$\begin{array}{l}\text { CGL2 C. elegans } \\
\quad N \text {-glycan } \\
\text { complex } / 2 \mathrm{WKK}\end{array}$} & \multirow{3}{*}[82,84]{} \\
\hline & & CGL3 chitotetraose complex/2R0H & 1.90 & & & \\
\hline & & & & 2R0F & & \\
\hline
\end{tabular}


Table 2. Cont.

\begin{tabular}{|c|c|c|c|c|c|c|}
\hline Lectin & Purification Strategy & $\begin{array}{l}\text { Description of Lectin/Lectin Complex and } \\
\text { Their PDB ID * }\end{array}$ & $\begin{array}{l}\text { Resolution } \\
\qquad(\AA)\end{array}$ & $\begin{array}{l}\text { Type of Fold }{ }^{\#} \text {, } \\
\text { PDB Structure and ID }\end{array}$ & $\begin{array}{c}\text { Similarity to Other } \\
\text { Structures and } \\
\text { Their PDB ID } \\
\end{array}$ & Ref. \\
\hline \multicolumn{7}{|l|}{$\beta$-Trefoil fold } \\
\hline \multirow{9}{*}{$\begin{array}{l}\text { Boletus edulis } \\
\text { lectin (BEL) }\end{array}$} & \multirow{9}{*}{$\begin{array}{l}\text { DEAE cellulose, } \\
\text { Superdex G75, MonoQ, } \\
\text { Lipidex } 1000\end{array}$} & BEL (Ligand free) form $1 / 4 \mathrm{I} 4 \mathrm{O}$ & 1.12 & \multirow{9}{*}{$4 \mathrm{I} 4 \mathrm{P}$} & \multirow{9}{*}{$\begin{array}{c}\text { LSL } \\
N- \\
\text { acetyllactoseamine } \\
\text { complex/1W3F }\end{array}$} & \multirow{9}{*}[177]{} \\
\hline & & BEL (Ligand free) form 2/4I4P & 1.28 & & & \\
\hline & & BEL (Ligand free) form 3/4I4Q & 1.51 & & & \\
\hline & & BEL (Ligand free) form $4 / 4 \mathrm{I} 4 \mathrm{R}$ & 1.77 & & & \\
\hline & & BEL lactose complex/4I4S & 1.40 & & & \\
\hline & & BEL galactose complex/4I4U & 1.57 & & & \\
\hline & & BEL $N$-acetylgalactosamine complex/4I4V & 1.50 & & & \\
\hline & & BEL T-Antigen disaccharide complex/4I4X & 1.72 & & & \\
\hline & & BEL T-Antigen complex/4I4Y & 1.90 & & & \\
\hline \multirow{4}{*}{$\begin{array}{l}\text { Clitocybe } \\
\text { nebularis lectin } \\
(\mathrm{CNL})\end{array}$} & \multirow{4}{*}{$\begin{array}{l}\text { Lactosyl and } \\
\text { glucosyl-sepharose AC, } \\
\text { Chromsep HPLC }\end{array}$} & CNL Lac complex at $\mathrm{pH} 4.4 / 3 \mathrm{NBC}$ & 1.01 & & \multirow{4}{*}{ Three Foil/3PG0 } & \multirow{4}{*}[79,81]{} \\
\hline & & CNL Lac complex at $\mathrm{pH} 7.1 / 3 \mathrm{NBD}$ & 1.15 & & & \\
\hline & & CNL $N, N^{N}$-diacetyllactosediamine complex/3NBE & 1.86 & & & \\
\hline & & & & $3 \mathrm{NBC}$ & & \\
\hline \multirow{3}{*}{$\begin{array}{l}\text { Coprinopsis } \\
\text { cinerea lectin } \\
\text { (CCL2) }\end{array}$} & & CCL2 (Ligand free)/2LIE & & & \multirow{3}{*}{$\begin{array}{l}\text { Mosquitocidal } \\
\text { toxin/2VSE }\end{array}$} & \multirow{3}{*}[83]{} \\
\hline & Horseradish Peroxidase AC & CCL2 nematode glycan complex/2LIQ & NA & & & \\
\hline & & & & 2LIE & & \\
\hline
\end{tabular}


Table 2. Cont

\begin{tabular}{|c|c|c|c|c|c|c|}
\hline Lectin & Purification Strategy & $\begin{array}{l}\text { Description of Lectin/Lectin Complex and } \\
\text { Their PDB ID * }\end{array}$ & $\begin{array}{l}\text { Resolution } \\
\qquad(\AA)\end{array}$ & $\begin{array}{l}\text { Type of Fold }{ }^{\#} \\
\text { PDB Structure and ID }\end{array}$ & $\begin{array}{c}\text { Similarity to Other } \\
\text { Structures and } \\
\text { Their PDB ID } \\
\end{array}$ & Ref. \\
\hline \multicolumn{7}{|l|}{$\beta$-Trefoil fold } \\
\hline \multirow{5}{*}{$\begin{array}{l}\text { Laetiporus } \\
\text { sulphureus } \\
\text { lectin (LSL) }\end{array}$} & \multirow{5}{*}{ Lactose-sepharose AC } & LSL (Ligand free)/1W3A & 2.65 & & \multirow{5}{*}{$\begin{array}{l}\text { Boletus edulis lectin } \\
\text { (BEL)/4I4O }\end{array}$} & \multirow{5}{*}[76,111,112]{} \\
\hline & & LSL $N$-acetyllactoseamine complex/1W3G & 2.68 & & & \\
\hline & & $\begin{array}{l}\text { LSL } N \text {-acetyllactoseamine complex in the } \\
\text { Gamma motif/1W3F }\end{array}$ & 2.58 & & & \\
\hline & & LSL (recombinant)/2Y9F & 1.47 & & & \\
\hline & & LSL (recombinant) Lac complex/2Y9G & 1.67 & $1 \mathrm{~W} 3 \mathrm{~A}$ & & \\
\hline \multirow{3}{*}{$\begin{array}{l}\text { Marasmius } \\
\text { oreades lectin } \\
\text { (MOA) }\end{array}$} & \multirow{3}{*}{$\begin{array}{l}\left(\mathrm{NH}_{4}\right)_{2} \mathrm{SO}_{4} \text { precipitation, } \\
\text { melibiose-sepharose, } \\
\text { Synsorb-type B trisaccharide, } \\
\text { and Synsorb-type A } \\
\text { trisaccharide AC }\end{array}$} & MOA Gal $\beta 1,3 \mathrm{Gal} \beta 1,4-\mathrm{GlcNAc}$ complex/2IHO & 2.41 & & \multirow{3}{*}{ Three Foil/3PG0 } & \multirow{3}{*}[122,178,179]{} \\
\hline & & MOA Gal $\alpha 1,3(F u c \alpha 1,2)$ Gal and calcium complex/3EF2 & 1.80 & & & \\
\hline & & & & $2 \mathrm{IHO}$ & & \\
\hline \multirow{6}{*}{$\begin{array}{l}\text { Macrolepiota } \\
\text { procera (MPL) }\end{array}$} & \multirow{6}{*}{ Lactosyl-Sepharose AC } & MPL (ligand free)/4ION & 1.60 & & \multirow{6}{*}{$\begin{array}{l}\text { Rhizoctonia solani } \\
\text { agglutinin/4G9M }\end{array}$} & \multirow{6}{*}[120]{} \\
\hline & & MPL Gal complex/4IYB & 1.59 & & & \\
\hline & & MPL Lac complex/4IZX & 1.1 & & & \\
\hline & & & & & & \\
\hline & & MPL $N$-acetyllactoseamine complex/4J2S & 1.4 & & & \\
\hline & & & & $4 \mathrm{ION}$ & & \\
\hline
\end{tabular}


Table 2. Cont.

\begin{tabular}{|c|c|c|c|c|c|c|}
\hline Lectin & Purification Strategy & $\begin{array}{l}\text { Description of Lectin/Lectin Complex and } \\
\text { Their PDB ID * }\end{array}$ & $\begin{array}{l}\text { Resolution } \\
\qquad(\AA ̊)\end{array}$ & $\begin{array}{l}\text { Type of Fold }{ }^{\#} \\
\text { PDB Structure and ID }\end{array}$ & $\begin{array}{c}\text { Similarity to Other } \\
\text { Structures and } \\
\text { Their PDB ID } \\
\end{array}$ & Ref. \\
\hline \multicolumn{7}{|l|}{$\beta$-Trefoil fold } \\
\hline \multirow[t]{2}{*}{$\begin{array}{l}\text { Polyporus } \\
\text { squamosus } \\
\text { lectin (PSL) }\end{array}$} & \multirow[t]{2}{*}{$\begin{array}{l}\left(\mathrm{NH}_{4}\right)_{2} \mathrm{SO}_{4} \text { precipitation, } \\
\beta \text {-D-galactosyl-Synsorb AC, } \\
\text { DEAE-Sephacel }\end{array}$} & \multirow[t]{2}{*}{$\begin{array}{l}\text { PSL bound to human-type influenza-binding epitope } \\
\text { Neu5Ac } \alpha 2-6 \mathrm{Gal} \beta 1-4 \mathrm{GlcNAc} / 3 \mathrm{PHZ}\end{array}$} & \multirow[t]{2}{*}{1.70} & & \multirow[t]{2}{*}{ ThreeFoil/3PG0 } & \multirow[t]{2}{*}[145,180]{} \\
\hline & & & & 3PHZ & & \\
\hline \multicolumn{7}{|c|}{ Actinoporin-like fold } \\
\hline \multirow{5}{*}{$\begin{array}{l}\text { Agaricus } \\
\text { bisporus lectin } \\
\text { (ABL) }\end{array}$} & \multirow{5}{*}{$\begin{array}{l}\text { Human erythrocytic stroma } \\
\text { polyacrylamide gel AC, } \\
\text { preparative isoelectric } \\
\text { focusing to separate the } \\
5 \mathrm{ABL} \text { isoforms }\end{array}$} & $\mathrm{ABL}$ (Ligand free)/1Y2T & 1.50 & \multirow{5}{*}{ 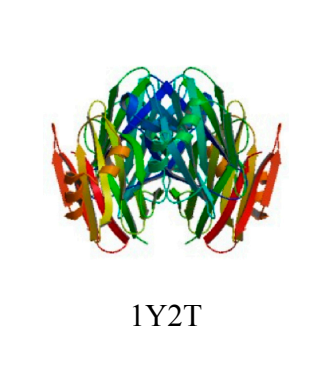 } & \multirow{5}{*}{$\begin{array}{c}\text { Sclerotium rolfsii } \\
\text { lectin }(\mathrm{SRL}) / 2 \mathrm{OFC}\end{array}$} & \multirow{5}{*}[51]{} \\
\hline & & ABL Lacto- $N$-biose complex/1Y2U & 1.85 & & & \\
\hline & & ABL T-antigen complex/1Y2V & 1.90 & & & \\
\hline & & $\begin{array}{l}\text { Orthorhombic form of ABL T-antigen and GlcNAc } \\
\text { complex/1Y2W }\end{array}$ & 1.74 & & & \\
\hline & & $\begin{array}{l}\text { Tetragonal form of ABL T-antigen and GlcNAc } \\
\text { complex/1Y2X }\end{array}$ & 2.36 & & & \\
\hline
\end{tabular}


Table 2. Cont

\begin{tabular}{|c|c|c|c|c|c|c|}
\hline Lectin & Purification Strategy & $\begin{array}{l}\text { Description of Lectin/Lectin Complex and } \\
\text { Their PDB ID * }\end{array}$ & $\begin{array}{l}\text { Resolution } \\
(\AA \AA)\end{array}$ & $\begin{array}{l}\text { Type of Fold }{ }^{\#} \text {, } \\
\text { PDB Structure and ID }\end{array}$ & $\begin{array}{c}\text { Similarity to Other } \\
\text { Structures and Their } \\
\text { PDB ID }\end{array}$ & Ref. \\
\hline Actinoporin-li & fold & & & & & \\
\hline \multirow{7}{*}{$\begin{array}{l}\text { Boletus edulis } \\
\text { lectin (BEL) }\end{array}$} & \multirow{7}{*}{$\begin{array}{l}\text { Chitin-sepharose AC, } \\
\text { Superdex G-200 HR, } \\
\text { Lipidex 1000; } \\
\text { Human erythrocytic stroma } \\
\text { polyacryl-amide gel AC, } \\
\text { Superdex G-200 HR, } \\
\text { Lipidex } 1000 \text { resin, } \\
\text { MiniQ PE }\end{array}$} & BEL (Ligand free)/3QDS & 1.15 & \multirow{7}{*}{ 3QDS } & \multirow{7}{*}{$\begin{array}{l}\text { Sclerotium rolfsii lectin } \\
(\mathrm{SRL}) / 2 \mathrm{OFC}\end{array}$} & \multirow{7}{*}[177]{} \\
\hline & & BEL T-antigen complex/3QDT & 1.30 & & & \\
\hline & & BEL $N, N$-diacetyl chitobiose complex/3QDU & 2.00 & & & \\
\hline & & $\begin{array}{l}\text { Orthorhombic form of BEL GlcNAc and } \\
\text { GalNAc complex/3QDV }\end{array}$ & 1.30 & & & \\
\hline & & $\begin{array}{l}\text { Hexagonal form of BEL GlcNAc and } \\
\text { GalNAc complex/3QDW }\end{array}$ & 1.90 & & & \\
\hline & & $\begin{array}{l}\text { Orthorhombic form of BEL T-antigen disaccharide } \\
\text { and } N, N \text {-diacetyl chitobiose complex/3QDX }\end{array}$ & 1.70 & & & \\
\hline & & $\begin{array}{l}\text { Hexagonal form of BEL T-antigen disaccharide } \\
\text { and } N, N \text {-diacetyl chitobiose complex/3QDY }\end{array}$ & 2.00 & & & \\
\hline \multirow{3}{*}{$\begin{array}{l}\text { Xerocomus } \\
\text { chrysenteron } \\
\text { lectin (XCL) }\end{array}$} & \multirow{3}{*}{ Fetuin-sepharose } & Wild-type XCL (ligand free)/1XI0 & 2.00 & & \multirow{3}{*}{$\begin{array}{l}\text { Sclerotium rolfsii lectin } \\
(\mathrm{SRL}) / 2 \mathrm{OFC}\end{array}$} & \multirow{3}{*}[107,181]{} \\
\hline & & XCL mutated at Q46M, V54M, L58M/1X99 & 1.40 & andis & & \\
\hline & & & & $1 \mathrm{XI} 0$ & & \\
\hline
\end{tabular}

* For further lectin-related tools and databases please see the Lectin 3D structure database (http://glyco3d.cermav.cnrs.fr) or the CAZy database (http://cazy.org);

$\wedge$ AC, Affinity Chromatography; ${ }^{\#}$ Only symmetrical, ligand free structures are shown; NA, Not Available. 
Table 3. Antiproliferative/antitumor and mitogenic activity of mushroom lectins.

\begin{tabular}{|c|c|c|c|}
\hline \multicolumn{4}{|c|}{ Antiproliferative/Antitumor Activity } \\
\hline Source of Lectin & $\mathrm{IC}_{50}$ & Cell Type/Target & Ref. \\
\hline Agaricus bisporus (ABL) & $50 \mu \mathrm{g} / \mathrm{mL} *$ & HT-29 & {$[50]$} \\
\hline Agrocybe aegerita (AAL galectin) & NA & $\begin{array}{l}\text { S-180, Hela, SW480,SGC 7901, } \\
\text { MGC80-3, BGC-823, HL-60 }\end{array}$ & {$[56]$} \\
\hline Amanita phalloides (A. phalloides lectin) & $1.7 \mu \mathrm{g} / \mathrm{mL} *$ & L1210 & {$[66]$} \\
\hline \multirow{3}{*}{ Armillaria luteovirens (ALL) } & $2.5 \mu \mathrm{M}$ & MBL2 & \multirow{3}{*}[69]{} \\
\hline & $5 \mu \mathrm{M}$ & $\mathrm{HeLa}$ & \\
\hline & $10 \mu \mathrm{M}$ & L1210 & \\
\hline Boletopsis leucomelaena (BLL) & $15 \mu \mathrm{g} / \mathrm{mL}$ & U937 & {$[182]$} \\
\hline Clitocybe nebularis (CNL) & NA & Mo-T, Jurkat & [79] \\
\hline Cordyceps militaris (CML) & $0.5-0.6 \mathrm{mg} / \mathrm{mL} *$ & HepG2 & {$[183]$} \\
\hline Flammulina velutipes ( $F$. velutipes lectin) & $13 \mu \mathrm{M}$ & $\mathrm{L} 1210$ & {$[86]$} \\
\hline \multirow{3}{*}{ Ganoderma capense (G. capense lectin) } & $8 \mu \mathrm{M}$ & L1210 & \multirow{3}{*}[88]{} \\
\hline & $16.5 \mu \mathrm{M}$ & HepG2 & \\
\hline & $12.5 \mu \mathrm{M}$ & M1 & \\
\hline Grifola frondosa (GFL) & $25 \mu \mathrm{g} / \mathrm{mL} *$ & $\mathrm{HeLa}$ & {$[184]$} \\
\hline \multirow{2}{*}{ Hericium erinaceus (HEA) } & $56.1 \mu \mathrm{M}$ & HepG2 & \multirow{2}{*}[94]{} \\
\hline & $76.5 \mu \mathrm{M}$ & MCF7 & \\
\hline \multirow{2}{*}{ Inocybe umbrinella (I. umbrinella lectin) } & $3.5 \mu \mathrm{M}$ & HepG2 & \multirow{2}{*}{ [99] } \\
\hline & $7.4 \mu \mathrm{M}$ & MCF7 & \\
\hline \multirow{3}{*}{ Lactarius flavidulus (LFL) } & $8.90 \mu \mathrm{M}$ & HepG2 & \multirow{3}{*}[106]{} \\
\hline & $6.81 \mu \mathrm{M}$ & L1210 & \\
\hline & $7.4 \mu \mathrm{M}$ & MCF7 & \\
\hline Paecilomyces japonica (PJA) & NA & SNU-1, AsPc-1, MDAMB-231 & {$[130]$} \\
\hline Paxillus involutus (P. involutus lectin) & NA & A-549, НCT-8 & {$[185]$} \\
\hline \multirow{2}{*}{ Pholiota adiposa (PAL) } & $2.1 \mu \mathrm{M}$ & HepG2 & \multirow{2}{*}[133]{} \\
\hline & $3.2 \mu \mathrm{M}$ & MCF7 & \\
\hline Pleurotus citrinopileatus (P. citrinopileatus lectin) & $5 \mathrm{mg} / \mathrm{kg}$ of body weight/day ${ }^{¥}$ & S-180 in ICR mice & [137] \\
\hline \multirow{2}{*}{ Pleurotus eous (PEL) } & $2 \mu \mathrm{g} / \mathrm{mL}$ & MCF-7, K562, HEP-2 & \multirow{2}{*}[139]{} \\
\hline & $50 \mu \mathrm{g} / \mathrm{mL}$ & SK-N-MC & \\
\hline Pleurotus ostreatus (POL) & $1.5 \mathrm{mg} / \mathrm{kg}$ bodyweight/day ${ }^{¥}$ & S-180, H-22 & {$[32]$} \\
\hline Polyporus adustus (P. adustus lectin) & NA & M1, Herto, S180 & {$[144]$} \\
\hline Psathyrella asperospora (PAL) & $0.48 \mu \mathrm{M}$ & HT29 & {$[186]$} \\
\hline \multirow{2}{*}{ Russula delica } & $0.88 \mu \mathrm{M}$ & HepG2 & \multirow{2}{*}[154]{} \\
\hline & $0.52 \mu \mathrm{M}$ & MCF 7 & \\
\hline Schizophyllum commune (SCL) & $30 \mu \mathrm{g} / \mathrm{mL}$ & $\mathrm{KB}$ & {$[156]$} \\
\hline Tricholoma mongolicum (TML1 \& TML2) & NA & P815, PU5-1.8 & {$[187]$} \\
\hline Volvariella volvacea (VVL) & $17.5 \mathrm{mg} / \mathrm{kg}$ body weight ${ }^{¥}$ & S-180 & {$[188]$} \\
\hline Xerocomus chrysenteron (XCL) & NA & Hela, NIH-3T3 & {$[189]$} \\
\hline \multirow{2}{*}{ Xylaria hypoxylon (X. hypoxylon lectin) } & $1.24 \mu \mathrm{M}$ & M1 & \multirow{2}{*}[162]{} \\
\hline & NA & HepG2 & \\
\hline
\end{tabular}


Table 3. Cont.

\begin{tabular}{|c|c|c|c|}
\hline \multicolumn{4}{|c|}{ Mitogenic Activity } \\
\hline Source of lectin & [Lectin] $^{\mathfrak{E}}$ & Cell type/Target & Ref. \\
\hline Agrocybe cylindracea (ACG) & $2 \mu \mathrm{M}$ & Mouse splenocytes & [61] \\
\hline Armillaria luteovirens (ALL) & $1 \mu \mathrm{M}$ & Mouse splenocytes & {$[69]$} \\
\hline Boletus edulis (BEL) & $1 \mu \mathrm{M}$ & Mouse splenocytes & {$[72]$} \\
\hline Cordyceps militaris (CML) & $26 \mu \mathrm{M}$ & Mouse splenocytes & {$[85]$} \\
\hline Flammulina velutipes ( $F$. velutipes lectin) & $100 \mu \mathrm{M}$ & Mouse spleen lymphocytes & {$[86,190]$} \\
\hline Hericium erinaceus (HEA) & $20 \mu \mathrm{M}$ & Mouse splenocytes & {$[94]$} \\
\hline Ganoderma capense (G. capense lectin) & $1.5 \mu \mathrm{M}$ & Mouse splenocytes & {$[88]$} \\
\hline Hygrophorus russula (HRL) & $0.15 \mu \mathrm{M}$ & Mouse splenocytes & {$[97]$} \\
\hline Peziza silvestris (P. silvestris lectin) & $8 \mu \mathrm{M}$ & Mouse splenocytes & {$[131]$} \\
\hline Pleurotus citrinopileatus (P. citrinopileatus lectin) & $2 \mu \mathrm{M}$ & Mouse splenocytes & {$[137]$} \\
\hline Polyporus adustus (P. adustus lectin) & $62.5 \mu \mathrm{M}$ & Mouse splenocytes & {$[144]$} \\
\hline Schizophyllum commune (SCL) & $4 \mu \mathrm{M}$ & Mouse splenocytes & {$[157]$} \\
\hline Xerocomus spadiceus (X. spadiceus lectin) & $31.25 \mu \mathrm{M}$ & Mouse splenocytes & {$[161]$} \\
\hline
\end{tabular}

NA: Not Available; * The concentration or range of titer found effective for antiproliferative/antitumor activities; ${ }^{¥}$ The dosage found effective for in vivo antiproliferative/antitumor activities; ${ }^{£}$ This is the minimum lectin concentration for mitogenic activity.

Table 4. Anti-HIV-1 reverse transcriptase activity of mushroom lectins.

\begin{tabular}{ccc}
\hline Source of Lectin & IC $_{\mathbf{5 0}}$ & Ref. \\
\hline Agaricus bisporus (ABL) & $8.0 \mu \mathrm{M}$ & {$[191]$} \\
Boletus edulis (BEL) & $14.3 \mu \mathrm{M}$ & {$[72]$} \\
Cordyceps militaris (CML) & $10.0 \mu \mathrm{M}$ & {$[183]$} \\
Hericium erinaceus (HEA) & $31.7 \mu \mathrm{M}$ & {$[94]$} \\
Inocybe umbrinella (I. umbrinella lectin) & $4.7 \mu \mathrm{M}$ & {$[99]$} \\
Pleurotus citrinopileatus (P. citrinopileatus lectin) & $0.93 \mu \mathrm{M}$ & {$[137]$} \\
Schizophyllum commune (SCL) & $1.2 \mu \mathrm{M}$ & {$[157]$} \\
\hline
\end{tabular}

\section{Mushroom Lectin Structures}

Mushroom lectins usually comprise two to four identical or non-identical subunits held together by non-covalent interactions. However, at least two examples exist of mushroom lectin subunits being linked by disulphide bridges (Lactarius lignyotus [107] and Phallus impudicus [192]). The molecular mass and oligomeric state of isolated mushroom lectins vary greatly, ranging from 10 to $190 \mathrm{kDa}$ [19]. Some notable examples include the tetrameric lectin with identical $16 \mathrm{kDa}$ subunits from Agaricus blazei [53], the 12.4 and $18 \mathrm{kDa}$ lectins isolated from the Ganoderma lucidum mycelia and fruiting bodies respectively [88], the $23 \mathrm{kDa}$ monomeric lectin from Auricularia polytricha [70], and the $114 \mathrm{kDa}$ hexameric lectin form Ganoderma capense [89,90]. The most commonly used methods for the purification of mushroom lectins are ion-exchange chromatography, affinity chromatography based on the sugar specificity of the lectin of interest, and size-exclusion chromatography, with the order and number of purification steps varying from lectin and lectin. Table 2 summarises the common strategies used to purify mushroom lectins for which crystal structure data exists. 
In order to better understand the atomic structure and molecular mechanisms underlying lectin-sugar interactions, it is necessary to study their crystallographic structure. The crystal structures of only 17 mushroom lectins have been determined (Table 2). A number of different structural families of mushroom lectins have been identified, including the ricin-like $\beta$-trefoil fold, galectin-like fold and actinoporin-like fold. The integrin-like seven-blade $\beta$-propeller and six-blade $\beta$-propeller as well as the actinoporin-like fold are distinctive for lectins from fungi [44,193]. Table 2 summarises the common structural characteristics mushroom lectins for which crystal structure data exists.

The crystal structures of two mushroom lectins have been reported to possess $\beta$-propeller-folds, the Aleuria aurantia lectin (AAL) and the Psathyrella velutina lectin (PVL). Among all mushroom lectins, PVL is the best studied. PVL is a multivalent and multi-substrate specific lectin that adopts a regular seven-bladed $\beta$-propeller-fold. The structure of PVL complexed with GlcNAc revealed six residues bound in pockets located between two consecutive propeller blades. A complex of PVL with Neu5Ac showed that the same hydrogen bond network as seen for GlcNAc are present, but the carbohydrate ring in the binding site is oriented differently [169]. The crystal structure of AAL complexed with Fuc revealed that each of the two monomers that make up AAL consist of a six-bladed $\beta$-propeller fold and a small antiparallel two-stranded $\beta$-sheet that is involved in dimerization. Interestingly, AAL was found to possess a multivalent carbohydrate recognition fold [166], similar to that seen in the Fuc binding lectin from Aspergillus fumigatus (AFL1), which has been proposed to be involved in the host pathogen interaction [194]. Structural PVL and AAL resemble $\alpha$-integrins, and are very similar to the bacterial lectin RSL from Ralstonia solanacearum that also adopts a $\beta$-propeller fold [195].

As previously mentioned a number of mushroom lectins have been found to adopt a galectin, or galectin-like fold. Galectins have a conserved carbohydrate recognition domain (CRD) that shares a $\beta$-Gal recognition pattern with highly conserved side chains. The galectins reported for Agrocybe aegerita (designated here AAL-galectin to distinguish it from the Aleuria aurantia lectin which is commonly referred to in the literature as AAL), Agrocybe cylindracea (ACG) and Coprinus cinereus (CGL2 and CGL3) belong to the proto-type galectin family. Based on sequence and structural comparison AAL-galectin and ACG are identical. The crystal structure of ACG complexed with Lac, 3'-sulfonyl Lac, and $\alpha 2,3$-sialyllactose revealed a $\beta$-sandwich structure of two antiparallel sheets, each with six strands, in contrast to the five and six strands in animal galectins [173]. Except for the substitution of one residue the $\mathrm{CRD}$ is the same as that seen in animal galectins. Interestingly, the presence of a 5-residue insertion in ACG alters the carbohydrate-binding site such that it is able to bind Neu5Ac [173]. The structure of CGL2 [175] and CGL3 [84] have also been studied complexed with a number of ligands including Lac, linear B2 trisaccharide, blood group A tetrasaccharide, and blood H Type II for CGL2, and chitotetraose (GlcNAc $\beta 1,4$ GlcNAc $\beta 1,4$ GlcNAc $\beta 1,4$ GlcNAc) for CGL3. CGL3 conserves all but one residue (Arg replaces Trp) known to be involved in Gal binding in galectins, resulting in CGL3 being unable to bind Lac. Instead CGL3 specifically and preferentially binds oligomers of GlcNAc. Interestingly, the mutation of Arg to Trp resulted in the lectin being unable to bind oligomers of GlcNAc and instead was able to bind Lac [84]. As will be discussed later in this review, a number of mushroom lectins have been found to have antitumour activity. One such example is AAL-galectin, which suppresses tumours through apoptosis-inducing activity in cancer cells [56]. The ThomsenFriedenreich antigen (TF antigen; Gal $\beta 1-3 \mathrm{GalNAc}-O$-Ser/Thr) is believed to be the ligand for AAL-galectin. The crystal structure of AAL-galectin complexed with the TF antigen revealed a unique recognition 
mode consisting of a hydrogen bond network formed by a conserved structural motif (Glu66-waterArg85-water) that provides new targets and opportunities for anticancer drug discovery [171].

Ricin B-like ( $\beta$-trefoil) lectins are carbohydrate-binding proteins similar to the B chain domains of ricin, a toxin from the castor bean (Ricinus communis) [196]. The main characteristic of these lectin domains is that they consist of three repeated subdomains, referred to as $\alpha-, \beta$ - and $\gamma$-repeats, each containing a well-conserved QXW motif [197]. Several ricin B-like lectins have been identified in mushrooms and crystal structures have been solved for Clitocybe nebularis lectin (CNL) [81], Laetiporus sulphureus lectin (LSL) [112], Marasmius oreades lectin (MOA) [178], Polyporus squamosus lectin (PSL) [180], Coprinopsis cinerea lectin (CCL2) [83], Macrolepiota procera (MPL) [120] and Boletus edulis lectin (BEL $\beta$-trefoil) [177]. LSL contains a pore-forming module [112], whereas a cysteine protease domain that also serves as a dimerization interface is present in MOA and PSL $[178,180,198]$. The toxic activities of these modular proteins have been attributed to their catalytic domains and the intracellular transport of the protein through binding to glycolipids or glycoproteins (Refer to Tables 1 and 2 for exact sugar specificity) that are facilitated by lectin domains $[198,199]$.

Another unique fold among the mushroom lectin is the actinoporin-like fold. The actinoporin-like fold consists of a $\beta$-sandwich made by two $\beta$-sheets composed of six and four $\beta$-strands respectively and connected by a helix-loop-helix motif. This fold has been found in Agaricus bisporus lectin (ABL) [51], Boletus edulis lectin (BEL) [177] and Xerocomus chrysenteron lectin (XCL) [181]. These lectins form dimers or tetramers that have two distinct binding sites per monomer that recognize the different configurations of a single epimeric hydroxyl.

Lyophyllum decastes lectin (LDL) is an interesting new addition to the known mushroom lectins. The recently resolved structure of LDL shows that this novel lectin adopts a unique lectin fold, where a core of two antiparallel $\beta$-sheets at the heart of a homodimer is connected to the periphery of the structure by intramolecular disulfide bridges [165]. Furthermore, the structure and LDL's fold suggests that it is an extracellular protein unlike most known mushroom lectins.

\section{Biological Activity of Mushroom Lectins}

\subsection{Antiproliferative/Antitumor Activity}

Tumour cell surfaces vary in composition of glycoconjugates in comparison to normal cells [200]. Lectins display antiproliferative potential by cross-linking these cell surface glycoconjugates or through immunomodulatory effects. The Gal $\beta 1,3 \mathrm{GalNAc-bindinglectin} \mathrm{from} \mathrm{the} \mathrm{edible} \mathrm{mushroom}$ Agaricus bisporus inhibits growth of colon cancer cells and breast cancer [50]. Similarly, the Volvariella volvacea lectin that has antiproliferative activity against Sarcomo S-180 cells [19], has also been shown to retard the growth of tumour cells in a mouse model, prolonging the life span of mice by $63 \%$ to $100 \%$. The lectin from Grifola frondosa is reported to be cytotoxic against HeLa cells at the lectin concentration of $25 \mu \mathrm{g} / \mathrm{mL}$ [184]. The antiproliferative activity of lectins has also been demonstrated in other mushroom species including Paxillus involutus [185], Lactarius flavidulus [106], Hericium erinaceus [94], Russula delica [154], Pholiota adiposa [133], and Clitocybe nebularis [79], and we have recently shown that the GlcNAc-specific lectin from Psathyrella asperospora (PAL) possess a potent antiproliferative activity (IC50: $0.48 \mu \mathrm{M})$ [186]. Further characterization of PAL's 
anti-proliferative activity showed that HT29 cells are arrested at G2/M phase of the cell cycle, and that this effect can be halted through the addition of free GlcNAc. Table 3 summarises the antiproliferative and antitumor activity of mushroom lectins along with their potent activity toward cancer cell lines.

\subsection{Mitogenic/Antimitogenic Activity}

Some lectins possess the remarkable property of stimulating the transformation of lymphocytes from small resting cells to large blast-like cells that may undergo mitosis [201]. Furthermore, the mechanism of this mitogenic activity, which involves activation and proliferation of lymphocytes, usually commences by binding of ligands to T-cell receptors, which triggers the signaling cascade, IL-2 gene expression and subsequent proliferation [202]. Lectins from Flammulina velutipes [190], Armillaria luteovirens [69], Ganoderma capense [88], Agrocybe cylindracea [61], Xerocomus spadiceus [161], Boletus edulis [72], Cordyceps militaris [85], Pleurotus citrinopileatus [137] and Hygrophorus russula [97] are known to be mitogenic with respect to murine splenocytes. In addition, the Volvariella volvacea lectin possesses mitogenic activity towards $\mathrm{T}$ lymphocytes through $\mathrm{T}$-cell receptor ensuing calcium signaling pathways [203]. The mitogenic potential of lectins frommushrooms is summarized in Table 3. However, lectins do not always display mitogenic activity to lymphocytes. Certain lectins have demonstrated antimitogenic activity. The lectin from Agaricus bisporus suppresses the activation of $\mathrm{T}$ and $\mathrm{B}$ lymphocytes [204]. Likewise, the lectins from Pleurotus flabellatus [203], Hericium erinaceus [203], Tricholoma mongolicum [45], Laetiporus sulphureus [113], Lactarius deliciosus [104], and Xylaria hypoxylon [162] are non-mitogenic.

\subsection{Immunomodulatory Activity}

There are only a few mushroom lectins that have been reported to regulate the components of the immune system. The lectins from Tricholoma mongolicum (TML-1 and TML-2) have been shown to activate macrophages through the generation of macrophage activating factor and tumor necrosis factor (TNF) in mice by stimulating the production of $\mathrm{NO}_{2}$ ions [187]. In a similar manner, $\mathrm{ABL}$ and ACG are also able to stimulate macrophage through the production of TNF- $\alpha$ and $\mathrm{NO}_{2}[205,206]$. Interestingly, ABL's immunomodulating activity was found to be thermal/freezing-resistant, acid/alkali tolerant and stable to dehydration making it a potential candidate as a stable immune stimulant in health foods and pharmaceuticals [205]. The lectin from Volvariella volvacea (VVL) also exerts a potent immunomodulatory effect in mice by inducing the gene expression of IL-2 and IFN- $\gamma$, thereby upregulating the Th-1 cell population [31]. In fact, VVL was nine-fold more potent than other non-lectin mushroom immunomodulating proteins in activating lymphocytes [207].

\subsection{Antiviral Activity}

The Paxillus involutus lectin possesses antiphytovirus activity towards tobacco mosaic virus with $70.6 \%$ inhibition at a concentration of $200 \mu \mathrm{g} / \mathrm{mL}$, however does nothave any inhibitory activity towards HIV-1 reverse transcriptase [185]. Hericium erinaceus agglutinin (HEA) on the other hand is a demonstrated inhibitor of HIV-1 reverse transcriptase activity with an IC50 of $31.7 \mu \mathrm{M}$ [94]. In fact a number of mushroom lectins havebeen found to havepotent anti-HIV-1 reverse transcriptase 
activity. For example, ABL, Schizophyllum commune lectin, BEL, Pleurotus citrinopileatus lectin, Cordyceps militaris lectin, and Inocybe umbrinella lectin, are all the inhibitors of HIV-1 reverse transcriptase [72,99,137,157,183,191]. The most potent anti-HIV-1 reverse transcriptase mushroom lectin yet identified comes from Pleurotus citrinopileatus (PCL) with an IC50 of $0.93 \mu \mathrm{M}$ [137].

The exact mechanism by which lectins in general exert their anti-HIV-1 reverse transcriptase activity is yet to be fully resolved but probably involves protein-protein interaction as demonstrated for the HIV-1 protease that also inhibits HIV-1 reverse transcriptase activity [208]. Besides mushroom lectins, antifungal proteins [209], ribosome inactivating proteins [210], and plant lectins [211] have also been shown to inhibit HIV-1 reverse transcriptase. Interestingly, the well characterized lectins Concanavalin A and ricin, and the red kidney bean agglutinin are potent inhibitors of DNA polymerase alpha activity, and DNA polymerase beta activity respectively [212]. The significance of this activity in relation to reverse transcriptase inhibition by other lectins awaits elucidation.

In addition to mushroom lectins, other fungal lectins also possess anti-HIV activity such as the cyanovirin- $N$ homologs (CVNHs) from Ceratopteris richardii and Neurospora crassa [213]. However these lectins were found not to be as effective as the native bacterial cyanovirin- $N$ and other known anti-viral lectins including griffithsin, scytovirin and microcystis virdis lectin [214]. The activity of cyanovirin- $N$ primarily arises from its association with the viral envelope glycoprotein 120 and to other cell surface receptors [215,216]. Again the significance and relevance of this mechanism in relation to anti-HIV activity shown by other lectins awaits elucidation.

\section{Conclusions and Future Perspectives}

The ability of lectins to bind specifically to glycoconjugates present on the cell surface has made them essential tools in diverse applications. For example, taxonomic study of protozoan parasites has been performed using the agglutinating extracts from several macrofungi [217,218]. In biomedical research, purified lectins are used to determine blood type due to the specificity of carbohydrate structures present on the cell surface of erythrocytes. Among the mushroom lectins, MOA has been reported to be specific for blood group B, while CGL2 is specific for blood group A tetrasaccharide [173,179]. Interestingly, the use of the Pleurotus ostreatus lectin (POL) as an adjuvant in hepatitis B virus (HBV) DNA vaccination has been reported to stimulate the immune response in transgenic mice. It has also been demonstrated that low dose of POL ( $1 \mu \mathrm{g} /$ mouse) in conjunction with HBV DNA vaccine has given stronger HBV-specific delayed-type hypersensitivity responses and higher HBV-specific IgG levels in that particular transgenic mice [219].

Another practical use of lectins is based on their immobilization to an inert chromatography support, which has allowed the isolation of particular membrane and serum glycoconjugates [40]. For example, AAL has been used to isolate different glycoconjugates, such as fucosylated glycoproteins from human erythrocyte membrane [220], brain glycoproteins [221], Bence-Jones proteins [222], tumour antigens [223], and human immunoglobulin G [224]. Furthermore, the specificity of lectins for sugars makes them valuable tools in glycobiology. For example, lectin arrays (immobilised lectins with known sugar binding specificity covalently immobilised onto glass microarray slides) are a relatively new tool in glycobiology that can be used to analyse the glycosylation pattern on the surface 
of cells, and, thus, determine its relevance in various cell processes including cell development and differentiation, cell-cell communication and pathogen-host recognition [225].

Despite the diversity of bioactivity attributed to lectins, there is only limited information available regarding the relationship between structure, function and biological activity of mushroom lectins. Recently, an attempt was made to structurally determine the correlation between tumour cell apoptosis-induction and Agrocybe aegerita lectin activity. It was reported that the prerequisite for tumour cell apoptosis inducedby Agrocybe aegerita lectin was the formation of dimers, and that binding of both Gal and Glc are required for lectin bioactivity [172]. Similarly, Pohleven et al. demonstrated that the carbohydrate binding and homodimerization properties of CNL, a ricin B-like lectin from Clitocybe nebularis, was essential for bioactivity, with non sugar-binding and nondimerizing monovalent CNL mutants being inactive [81]. Further studies are required to determine the mechanism of action that gives mushroom lectins, and lectins from other sources, their diverse and potent bioactivity.

The potential use of mushroom lectins in therapy will also require the large-scale production of pure, fully functioning protein. Currently the majority of potentially medical useful mushroom lectins are purified from fruit-bodies collected in nature. This not only gives low yields, but is also time-consuming and expensive, and can also lead to batch variation. Moreover, the native isolation of lectins from mushrooms can lead to batch variation, due to environmental influences such as season, location and year of harvest, as well as differences in mushroom maturity and mycelia growth $[7,40]$. Therefore there is a need to advance efforts to clone and recombinantly express functional lectins. Over the past decade, only a small number of mushroom lectins have been expressed in Escherichia coli. For example, AAL and AAL2 lectin from the edible mushroom Agrocybe aegerita have been cloned and functionally expressed in E. coli [59,172]. In addition, CNL [81], MOA [178], XCL [181], and PSL [180] have also all been expressed in E. coli, and CGL2 has been expressed in the yeast Saccharomyces cerevisiae [175]. However, the majority of these lectins have been recombinantly expressed to obtain lectin in high enough yields for crystal structure determination. Recombinant lectin production for potential therapeutic use is still in its infancy, and will require further research and development particularly with regard to the importance and requirements for post-translational modifications of lectins for therapeutic use in humans.

\section{Author Contributions}

Mohamed Ali Abol Hassan and Razina Rouf reviewed the relevant literature, prepared the tables and wrote the first draft of the manuscript. Evelin Tiralongo and Tom W. May read, revised the text and co-wrote the manuscript. Joe Tiralongo supervised and co-wrote the review, revised the text and tables, and prepared the manuscript for submission.

\section{Conflicts of Interest}

The authors declare no conflicts of interest. 


\section{References}

1. Sharon, N.; Lis, H. Lectins as cell recognition molecules. Science 1989, 246, 227-234.

2. Kocourek, J.; Horejsi, V. A note on the recent discussion on definition of the term "Lectin". Lectins Biol. Biochem. Clin. Biochem. 1983, 3, 3-6.

3. Ashwell, G.; Harford, J. Carbohydrate-specific receptors of the liver. Annu. Rev. Biochem. 1982, 51, 531-554.

4. $\quad$ Springer, T.A.; Lasky, L.A. Sticky sugars for selectins. Nature 1991, 349, 196-197.

5. Cash, H.L.; Whitham, C.V.; Behrendt, C.L.; Hooper, L.V. Symbiotic bacteria direct expression of an intestinal bactericidal lectin. Science 2006, 313, 1126-1130.

6. Imberty, A.; Gautier, C.; Lescar, J.; Pérez, S.; Wyns, L.; Loris, R. An unusual carbohydrate binding site revealed by the structures of two Maackia amurensis lectins complexed with sialic acid-containing oligosaccharides. J. Biol. Chem. 2000, 275, 17541-17548.

7. Rouf, R.; Tiralongo, E.; Krahl, A.; Maes, K.; Spaan, L.; Wolf, S.; May, T.W.; Tiralongo, J. Comparative study of hemagglutination and lectin activity in Australian medicinal mushrooms (higher Basidiomycetes). Int. J. Med. Mushrooms 2011, 13, 493-504.

8. Singh, R.S.; Tiwary, A.K.; Kennedy, J.F. Lectins: Sources, activities, and applications. Crit. Rev. Biotechnol. 1999, 19, 145-178.

9. Wright, L.M.; van Damme, E.J.; Barre, A.; Allen, A.K.; van Leuven, F.; Reynolds, C.D.; Rouge, P.; Peumans, W.J. Isolation, characterization, molecular cloning and molecular modelling of two lectins of different specificities from bluebell (Scilla campanulata) bulbs. Biochem. J. 1999, 340 Pt 1, 299-308.

10. Wright, L.M.; Wood, S.D.; Reynolds, C.D.; Rizkallah, P.J.; Peumans, W.J.; van Damme, E.J.; Allen, A.K. Purification, crystallization and preliminary X-ray analysis of a mannose-binding lectin from bluebell (Scilla campanulata) bulbs. Acta Crystallogr. D 1996, 52, 1021-1023.

11. Van Damme, E.J.; Balzarini, J.; Smeets, K.; van Leuven, F.; Peumans, W.J. The monomeric and dimeric mannose-binding proteins from the Orchidaceae species Listera ovata and Epipactis helleborine: Sequence homologies and differences in biological activities. Glycoconj. J. 1994, 11, $321-332$.

12. Van Damme, J.M.; Smeets, K.; Torrekens, S.; van Leuven, F.; Peumans, W.J. Characterization and molecular cloning of mannose-binding lectins from the Orchidaceae species Listera ovata, Epipactis helleborine and Cymbidium hybrid. Eur. J. Biochem. 1994, 221, 769-777.

13. Van Damme, E.J.; Barre, A.; Mazard, A.M.; Verhaert, P.; Horman, A.; Debray, H.; Rouge, P.; Peumans, W.J. Characterization and molecular cloning of the lectin from Helianthus tuberosus. Eur. J. Biochem. 1999, 259, 135-142.

14. Lis, H.; Sharon, N. Lectins: Carbohydrate-Specific proteins that mediate cellular recognition. Chem. Rev. 1998, 98, 637-674.

15. Gatehouse, A.M.; Powell, K.S.; Peumans, W.J.; van Damme, E.J.; Gatehouse, J.A. Insecticidal properties of plant lectins: Their potential in plant protection. In Lectins Biomedical Perspectives; Taylor \& Francis e-library: Bristol, PA, USA, 1995; pp. 35-57.

16. Brock, T.; Madigan, M.; Martinko, J.; Parker, J. Biology of Microorganisms, 7th ed.; Prentice-Hall International, Inc.: Englewood Cliffs, NJ, USA, 1994. 
17. Varki, A.; Cummings, R.; Esko, J.; Freeze, H.; Hart, G.; Marth, J. Structures common to different types of glycans. In Essentials of Glycobiology; Cold Spring Harbor Laboraory Press: New York, NY, USA, 1999; pp. 211-252.

18. Sharon, N. Lectin receptors as lymphocyte surface markers. Adv. Immunol. 1983, 34, 213-298.

19. Wang, H.; Ng, T.B.; Ooi, V.E.C. Lectins from mushrooms. Mycol. Res. 1998, 102, 897-906.

20. Tiwary, A.; Singh, R. Lectins: Novel drug targeting molecules. Indian J. Pharm. Sci. 1999, 61, 259.

21. Chang, S.T.; Buswell, J. Mushroom nutriceuticals. World J. Microbiol. Biotechnol. 1996, 12, 473-476.

22. Wasser, S.P.; Sokolov, D.; Reshetnikov, S.V.; Timor-Tismenetsky, M. Dietary supplements from medicinal mushrooms: Diversity of types and variety of regulations. Int. J. Med. Mushrooms 2000, 2, 1-19.

23. Breene, W.M. Nutritional and medicinal value of specialty mushrooms. J. Food Prot. 1990, 53, 883-894.

24. Chang, S.T.; Miles, P.G. Mushrooms biology-a new discipline. Mycologist 1992, 6, 64-65.

25. Hibbett, D.S.; Binder, M.; Bischoff, J.F.; Blackwell, M.; Cannon, P.F.; Eriksson, O.E.; Huhndorf, S.; James, T.; Kirk, P.M.; Lücking, R. A higher-level phylogenetic classification of the Fungi. Mycol. Res. 2007, 111, 509-547.

26. Lindequist, U.; Niedermeyer, T.H.; Jülich, W.-D. The pharmacological potential of mushrooms. Evid.-Based Complement. Altern. Med. 2005, 2, 285-299.

27. Wasser, S.P. Current findings, future trends, and unsolved problems in studies of medicinal mushrooms. Appl. Microbiol. Biotechnol. 2011, 89, 1323-1332.

28. Wasser, S.P. Medicinal mushrooms as a source of antitumor and immunomodulating polysaccharides. Appl. Microbiol. Biotechnol. 2002, 60, 258-274.

29. Hawksworth, D.L. Mushrooms: The extent of the unexplored potential. Int. J. Med. Mushrooms 2001, 3, 333-340.

30. Erjavec, J.; Kos, J.; Ravnikar, M.; Dreo, T.; Sabotič, J. Proteins of higher fungi-From forest to application. Trends Biotechnol. 2012, 30, 259-273.

31. She, Q.B.; Ng, T.B.; Liu, W.K. A novel lectin with potent immunomodulatory activity isolated from both fruiting bodies and cultured mycelia of the edible mushroom Volvariella volvacea. Biochem. Biophys. Res. Commun. 1998, 247, 106-111.

32. Wang, H.; Gao, J.; Ng, T.B. A new lectin with highly potent antihepatoma and antisarcoma activities from the oyster mushroom Pleurotus ostreatus. Biochem. Biophys. Res. Commun. 2000, $275,810-816$.

33. Weis, A.L.; Wasser, S.P. Therapeutic effects of substances occurring in higher basidiomycetes mushrooms: A modern perspective. Crit. Rev. Immunol. 1999, 19, 32.

34. Sliva, D. Ganoderma lucidum in cancer research. Leuk. Res. 2006, 30, 767-768.

35. Kim, B.K.; Cho, H.Y.; Kim, J.S.; Kim, H.W.; Choi, E.C. Studies on constituents of higher Fungi of Korea (LXVIII). Studies 1993, 24, 203-212.

36. Singh, R.S.; Bhari, R.; Kaur, H.P. Mushroom lectins: Current status and future perspectives. Crit. Rev. Biotechnol. 2010, 30, 99-126.

37. Khan, F.; Khan, M.I. Fungal lectins: Current molecular and biochemical perspectives. Int. J. Biol. Chem. 2011, 5, 1-20. 
38. Sharon, N.; Lis, H. History of lectins: From hemagglutinins to biological recognition molecules. Glycobiology 2004, 14, 53R-62R.

39. Końska, G. The lectins of higher fungi (macromycetes) - Their occurrence, physiological role and biological activity. Int. J. Med. Mushrooms 2006, 8, 19-30.

40. Guillot, J.; Konska, G. Lectins in higher fungi. Biochem. Syst. Ecol. 1997, 25, 203-230.

41. Singh, S.S.; Wang, H.; Chan, Y.S.; Pan, W.; Dan, X.; Yin, C.M.; Akkouh, O.; Ng, T.B. Lectins from Edible Mushrooms. Molecules 2014, 20, 446-469.

42. Peumans, W.J.; van Damme, E.J. Lectins as plant defense proteins. Plant Physiol. 1995, 109, $347-352$.

43. Swamy, B.M.; Bhat, A.G.; Hegde, G.V.; Naik, R.S.; Kulkarni, S.; Inamdar, S.R. Immunolocalization and functional role of Sclerotium rolfsii lectin in development of fungus by interaction with its endogenous receptor. Glycobiology 2004, 14, 951-957.

44. Varrot, A.; Basheer, S.M.; Imberty, A. Fungal lectins: Structure, function and potential applications. Curr. Opin. Struct. Biol. 2013, 23, 678-685.

45. Wang, H.X.; Ng, T.B.; Liu, W.K.; Ooi, V.E.C.; Chang, S.T. Isolation and characterization of two distinct lectins with antiproliferative activity from the cultured mycelium of the edible mushroom Tricholoma mongolicum. Int. J. Pept. Protein Res. 1995, 46, 508-513.

46. Kawagishi, H.; Mitsunaga, S.; Yamawaki, M.; Ido, M.; Shimada, A.; Kinoshita, T.; Murata, T.; Usui, T.; Kimura, A.; Chiba, S. A lectin from mycelia of the fungus Ganoderma lucidum. Phytochemistry 1997, 44, 7-10.

47. Mikiashvili, N.; Elisashvili, V.; Wasser, S.P.; Nevo, E. Comparative study of lectin activity of higher Basidiomycetes. Int. J. Med. Mushrooms 2006, 8, 31-38.

48. Zhao, J.K.; Zhao, Y.C.; Li, S.H.; Wang, H.X.; Ng, T.B. Isolation and characterization of a novel thermostable lectin from the wild edible mushroom Agaricus arvensis. J. Basic Microbiol. 2011, 51, 304-311.

49. Nakamura-Tsuruta, S.; Kominami, J.; Kuno, A.; Hirabayashi, J. Evidence that Agaricus bisporus agglutinin (ABA) has dual sugar-binding specificity. Biochem. Biophys. Res. Commun. 2006, 347, 215-220.

50. Yu, L.; Fernig, D.G.; Smith, J.A.; Milton, J.D.; Rhodes, J.M. Reversible inhibition of proliferation of epithelial cell lines by Agaricus bisporus (edible mushroom) lectin. Cancer Res. 1993, 53, 4627-4632.

51. Carrizo, M.E.; Capaldi, S.; Perduca, M.; Irazoqui, F.J.; Nores, G.A.; Monaco, H.L. The antineoplastic lectin of the common edible mushroom (Agaricus bisporus) has two binding sites, each specific for a different configuration at a single epimeric hydroxyl. J. Biol. Chem. 2005, 280, 10614-10623.

52. Batterbury, M.; Tebbs, C.A.; Rhodes, J.M.; Grierson, I. Agaricus bisporus (edible mushroom lectin) inhibits ocular fibroblast proliferation and collagen lattice contraction. Exp. Eye Res. 2002, 74, 361-370.

53. Kawagishi, H.; Aya, N.; Takayuki, Y.; Takashi, M.; Toshihiko, H.; Takuji, N. Isolation and properties of a lectin from the fruiting bodies of Agaricus blazei. Carbohydr. Res. 1988, 183, $150-154$.

54. Sage, H.J.; Vazquez, J.J. Studies on a hemagglutinin from the mushroom agaricus campestris. J. Biol. Chem. 1967, 242, 120-125. 
55. Sage, H.J.; Connett, S.L. Studies on a hemagglutinin from the meadow mushroom: II. purification, composition, and structure of agaricus campestris hemagglutinin. J. Biol. Chem. 1969, 244, 4713-4719.

56. Zhao, C.; Sun, H.; Tong, X.; Qi, Y. An antitumour lectin from the edible mushroom Agrocybe aegerita. Biochem. J. 2003, 374, 321-327.

57. Yang, N.; Liang, Y.; Xiang, Y.; Zhang, Y.; Sun, H.; Wang, D.C. Crystallization and preliminary crystallographic studies of an antitumour lectin from the edible mushroom Agrocybe aegerita. Protein Pept. Lett. 2005, 12, 705-707.

58. Sun, H.; Zhao, C.G.; Tong, X.; Qi, Y.P. A lectin with mycelia differentiation and antiphytovirus activities from the edible mushroom Agrocybe aegerita. J. Biochem. Mol. Biol. 2003, 36, 214-222.

59. Jiang, S.; Chen, Y.; Wang, M.; Yin, Y.; Pan, Y.; Gu, B.; Yu, G.; Li, Y.; Wong, B.H.; Liang, Y.; et al. A novel lectin from Agrocybe aegerita shows high binding selectivity for terminal $\mathrm{N}$-acetylglucosamine. Biochem. J. 2012, 443, 369-378.

60. Yagi, F.; Miyamoto, M.; Abe, T.; Minami, Y.; Tadera, K.; Goldstein, I.J. Purification and carbohydrate-binding specificity of Agrocybe cylindracea lectin. Glycoconj. J. 1997, 14, 281-288.

61. Wang, H.; Ng, T.B.; Liu, Q. Isolation of a new heterodimeric lectin with mitogenic activity from fruiting bodies of the mushroom Agrocybe cylindracea. Life Sci. 2002, 70, 877-885.

62. Lehmann, F.; Tiralongo, E.; Tiralongo, J. Sialic acid-specific lectins: Occurrence, specificity and function. Cell. Mol. Life Sci. 2006, 63, 1331-1354.

63. Yagi, F.; Hiroyama, H.; Kodama, S. Agrocybe cylindracea lectin is a member of the galectin family. Glycoconj. J. 2001, 18, 745-749.

64. Kochibe, N.; Furukawa, K. Purification and properties of a novel fucose-specific hemagglutinin of Aleuria aurantia. Biochemistry 1980, 19, 2841-2846.

65. Olausson, J.; Tibell, L.; Jonsson, B.H.; Pahlsson, P. Detection of a high affinity binding site in recombinant Aleuria aurantia lectin. Glycoconj. J. 2008, 25, 753-762.

66. Lutsik-Kordovsky, M.D.; Stasyk, T.V.; Stoika, R.S. Analysis of cytotoxicity of lectin and non-lectin proteins from Amanita mushrooms. Eksp. Onkol. 2001, 23, 43-45.

67. Zhuang, C.; Murata, T.; Usui, T.; Kawagishi, H.; Kobayashi, K. Purification and characterization of a lectin from the toxic mushroom Amanita pantherina. Biochim. Biophys. Acta Gen. Subj. 1996, 1291, 40-44.

68. Antonyuk, V.O.; Yu Klyuchivska, O.; Stoika, R.S. Cytotoxic proteins of Amanita virosa Secr. mushroom: Purification, characteristics and action towards mammalian cells. Toxicon 2010, 55, 1297-1305.

69. Feng, K.; Liu, Q.H.; Ng, T.B.; Liu, H.Z.; Li, J.Q.; Chen, G.; Sheng, H.Y.; Xie, Z.L.; Wang, H.X. Isolation and characterization of a novel lectin from the mushroom Armillaria luteo-virens. Biochem. Biophys. Res. Commun. 2006, 345, 1573-1578.

70. Yagi, F.; Tadera, K. Purification and characterization of lectin from Auricularia polytricha. Agric. Biol. Chem. 1988, 52, 2077-2079.

71. Koyama, Y.; Suzuki, T.; Odani, S.; Nakamura, S.; Kominami, J.; Hirabayashi, J.; Isemura, M. Carbohydrate specificity of lectins from Boletopsis leucomelas and Aralia cordate. Biosci. Biotechnol. Biochem. 2006, 70, 542-545. 
72. Zheng, S.; Li, C.; Ng, T.B.; Wang, H.X. A lectin with mitogenic activity from the edible wild mushroom Boletus edulis. Process Biochem. 2007, 42, 1620-1624.

73. Licastro, F.; Morini, M.C.; Kretz, O.; Dirheimer, G.; Creppy, E.E.; Stirpe, F. Mitogenic activity and immunological properties of bolesatine, a lectin isolated from the mushroom Boletus satanas Lenz. Int. J. Biochem. 1993, 25, 789-792.

74. Colceag, J.; Mogos, S.; Hulea, S. Studies on occurrence and characterization of phytolectins in some species of mushrooms. Rev. Roum. Biochim. 1984, 21, 263-266.

75. Horibe, M.; Kobayashi, Y.; Dohra, H.; Morita, T.; Murata, T.; Usui, T.; Nakamura-Tsuruta, S.; Kamei, M.; Hirabayashi, J.; Matsuura, M.; et al. Toxic isolectins from the mushroom Boletus venenatus. Phytochemistry 2010, 71, 648-657.

76. Kobayashi, Y.; Ishizaki, T.; Kawagishi, H. Screening for lectins in wild and cultivated mushrooms from Japan and their sugar-binding specificities. Int. J. Med. Mushrooms 2004, 6, $117-129$.

77. Otta, Y.; Amano, K.; Nishiyama, K.; Ando, A.; Ogawa, S.; Nagata, Y. Purification and properties of a lectin from ascomycete mushroom, Ciborinia camelliae. Phytochemistry 2002, 60, 103-107.

78. Lyimo, B.; Funakuma, N.; Minami, Y.; Yagi, F. Characterization of a new alpha-Galactosyl-Binding Lectin from the Mushroom Clavaria purpurea. Biosci. Biotechnol. Biochem. 2012, 76, 336-342.

79. Pohleven, J.; Obermajer, N.; Sabotic, J.; Anzlovar, S.; Sepcic, K.; Kos, J.; Kralj, B.; Strukelj, B.; Brzin, J. Purification, characterization and cloning of a ricin B-like lectin from mushroom Clitocybe nebularis with antiproliferative activity against human leukemic $\mathrm{T}$ cells. Biochim. Biophys. Acta 2009, 1790, 173-181.

80. Horejsi, V.; Kocourek, J. Studies on lectins. XXXVI. Properties of some lectins prepared by affinity chromatography on $O$-glycosyl polyacrylamide gels. Biochim. Biophys. Acta 1978, 538, 299-315.

81. Pohleven, J.; Renko, M.; Magister, Š.; Smith, D.F.; Künzler, M.; Štrukelj, B.; Turk, D.; Kos, J.; Sabotič, J. Bivalent carbohydrate binding is required for biological activity of Clitocybe nebularis Lectin (CNL), the N,N'-Diacetyllactosediamine (GalNAc $\beta 1-4 G l c N A c$, LacdiNAc)-specific Lectin from Basidiomycete C. nebularis. J. Biol. Chem. 2012, 287, 10602-10612.

82. Cooper, D.N.; Boulianne, R.P.; Charlton, S.; Farrell, E.M.; Sucher, A.; Lu, B.C. Fungal galectins, sequence and specificity of two isolectins from Coprinus cinereus. J. Biol. Chem. 1997, 272, $1514-1521$.

83. Schubert, M.; Bleuler-Martinez, S.; Butschi, A.; Walti, M.A.; Egloff, P.; Stutz, K.; Yan, S.; Collot, M.; Mallet, J.M.; Wilson, I.B.; et al. Plasticity of the beta-trefoil protein fold in the recognition and control of invertebrate predators and parasites by a fungal defence system. PLoS Pathog. 2012, 8, e1002706.

84. Walti, M.A.; Walser, P.J.; Thore, S.; Grunler, A.; Bednar, M.; Kunzler, M.; Aebi, M. Structural basis for chitotetraose coordination by CGL3, a novel galectin-related protein from Coprinopsis cinerea. J. Mol. Biol. 2008, 379, 146-159.

85. Jung, E.C.; Kim, K.D.; Bae, C.H.; Kim, J.C.; Kim, D.K.; Kim, H.H. A mushroom lectin from ascomycete Cordyceps militaris. Biochim. Biophys. Acta 2007, 1770, 833-838.

86. Ng, T.B.; Ngai, P.H.; Xia, L. An agglutinin with mitogenic and antiproliferative activities from the mushroom Flammulina velutipes. Mycologia 2006, 98, 167-171. 
87. Yatohgo, T.; Nakata, M.; Tsumuraya, Y.; Hashimoto, Y.; Yamamoto, S. Purification and properties of a lectin from the fruitbodies of Flammulina velutipes. Agric. Biol. Chem. 1988, 52, 1485-1493.

88. Ngai, P.H.K.; Ng, T.B. A mushroom (Ganoderma capense) lectin with spectacular thermostability, potent mitogenic activity on splenocytes, and antiproliferative activity toward tumor cells. Biochem. Biophys. Res. Commun. 2004, 314, 988-993.

89. Thakur, A.; Rana, M.; Lakhanpal, T.N.; Ahmad, A.; Khan, M.I. Purification and characterization of lectin from fruiting body of Ganoderma lucidum. Biochim. Biophys. Acta Gen. Subj. 2007, 1770, 1404-1412.

90. Thakur, A.; Pal, L.; Ahmad, A.; Khan, M.I. Complex carbohydrate specificity of lectin from fruiting body of Ganoderma lucidum. A surface plasmon resonance study. IUBMB Life 2007, 59, $758-764$.

91. Nagata, Y.; Yamashita, M.; Honda, H.; Akabane, J.; Uehara, K.; Saito, A.; Sumisa, F.; Nishibori, K.; Oodaira, Y. Characterization, occurrence, and molecular cloning of a lectin from Grifola frondosa: Jacalin-related lectin of fungal origin. Biosci. Biotechnol. Biochem. 2005, 69, 2374-2380.

92. Stepanova, L.; Burygin, G.; Matora, L.; Bogatyrev, V.; Sokolova, M.; Nikitina, V. Localization and immunochemical characteristics of basidiomycete Grifola frondosa (Fr.) SF Gray lectin. Mikrobiologiia 2009, 78, 236.

93. Kawagishi, H.; Mori, H.; Uno, A.; Kimura, A.; Chiba, S. A sialic acid-binding lectin from the mushroom Hericium erinaceum. FEBS Lett. 1994, 340, 56-58.

94. Li, Y.; Zhang, G.; Ng, T.B.; Wang, H. A novel lectin with antiproliferative and HIV-1 reverse transcriptase inhibitory activities from dried fruiting bodies of the monkey head mushroom Hericium erinaceum. J. Biomed. Biotechnol. 2010, 2010, 716515.

95. Veau, B.; Guillot, J.; Damez, M.; Dusser, M.; Konska, G.; Botton, B. Purification and characterization of an anti-(A+B) specific lectin from the mushroom Hygrophorus hypothejus. Biochim. Biophys. Acta 1999, 1428, 39-44.

96. Guillot, J.; Coulet, M. Properties of the anti (A plus B) lectin of Hygrophorus hypothejus Fr. Fixation, elution, inhibition. Rev. Fr. Transfus. 1974, 17, 49.

97. Suzuki, T.; Sugiyama, K.; Hirai, H.; Ito, H.; Morita, T.; Dohra, H.; Murata, T.; Usui, T.; Tateno, H.; Hirabayashi, J.; et al. Mannose-specific lectin from the mushroom Hygrophorus russula. Glycobiology 2012, 22, 616-629.

98. Coulet, M.; Guillot, J.; Betail, G. Action of certain mono- and polysaccharides on hemagglutinins of certain mushrooms. Acta Pol. Pharm. 1972, 29, 299-307.

99. Zhao, J.K.; Wang, H.X.; Ng, T.B. Purification and characterization of a novel lectin from the toxic wild mushroom Inocybe umbrinella. Toxicon 2009, 53, 360-366.

100. Musflek, M.; Ticha, M.; Volc, J.; Kocourek, J. Studies on lectins LXXI. Lectins in mycelial cultures of Kuehneromyces mutabilis, Pholiota squarrosa, and Flammulina velutipes. In Proceedings of the Tenth Lectin Meeting, Prague, Czech Republic, July 1988; Sigma Aldrich Corp: St. Louis, MI, USA, 1990; p. 53.

101. Guillot, J.; Genaud, L.; Gueugnot, J.; Damez, M. Purification and properties of two hemagglutinins of the mushroom Laccaria amethystina. Biochemistry 1983, 22, 5365-5369. 
102. Lyimo, B.; Yagi, F.; Minami, Y. Primary structure and specificity of a new member of galectin family from the Amethyst deceiver mushroom Laccaria amethystina. Biosci. Biotechnol. Biochem. 2011, 75, 62-69.

103. Ticha, M.; Sychrova, H.; Kocourek, J. Saccharide binding properties of lectins of higher fungi. In Lectins; Bøg-Hansen, T.C., Freed, D.L.J., Eds.; Sigma chemical company: St. Louis, MI, USA, 1988; Volume 6, pp. 383-391.

104. Guillot, J.; Giollant, M.; Damez, M.; Dusser, M. Isolation and characterization of a lectin from the mushroom, Lactarius deliciosus. J. Biochem. 1991, 109, 840-845.

105. Giollant, M.; Guillot, J.; Damez, M.; Dusser, M.; Didier, P.; Didier, E. Characterization of a lectin from Lactarius deterrimus. Research on the possible involvement of the fungal lectin in recognition between mushroom and spruce during the early stages of mycorrhizae formation. Plant Physiol. 1993, 101, 513-522.

106. Wu, Y.; Wang, H.; Ng, T.B. Purification and characterization of a lectin with antiproliferative activity toward cancer cells from the dried fruit bodies of Lactarius flavidulus. Carbohydr. Res. 2011, 346, 2576-2581.

107. Sychrova, H.; Ticha, M.; Kocourek, J. Studies on lectins. LIX. Isolation and properties of lectins from fruiting bodies of Xerocomus chrysenteron and Lactarius lignyotus. Can. J. Biochem. Cell Biol. 1985, 63, 700-704.

108. Panchak, L.V.; Antonyuk, V.O. Purification of a lectin from fruit bodies of Lactarius pergamenus (Fr.) Fr. and studies of its properties. Biochemistry 2011, 76, 438-449.

109. Panchak, L.V.; Antoniuk, V.O. Purification of lectin from fruiting bodies of Lactarius rufus (Scop.: Fr.)Fr. and its carbohydrate specificity. Ukr. Biokhim. Zhurnal 2007, 79, 123-128.

110. Giollant, M. Les Lectines des Lactaires du Groupe Dapestes: (L. deiciosus, L. deterrimus, L. salmonicolor). Purification, Étude Biochimique et Spécificité. Invervention des Lectines dans les Phénoménes de Reconnaissance Moléculaire au Cours des Événements Précoces de la Mycorrhization avec les Conifers Associés. Thése de Doctorat en Pharmacie, Univ. Clermont I, Clermont-Ferrand, France, 1991.

111. Tateno, H.; Goldstein, I.J. Molecular cloning, expression, and characterization of novel hemolytic lectins from the mushroom Laetiporus sulphureus, which show homology to bacterial toxins. J. Biol. Chem. 2003, 278, 40455-40463.

112. Mancheno, J.M.; Tateno, H.; Goldstein, I.J.; Martinez-Ripoll, M.; Hermoso, J.A. Structural analysis of the Laetiporus sulphureus hemolytic pore-forming lectin in complex with sugars. J. Biol. Chem. 2005, 280, 17251-17259.

113. Konska, G.; Guillot, J.; Dusser, M.; Damez, M.; Botton, B. Isolation and characterization of an $\mathrm{N}$-acetyllactosamine-binding lectin from the mushroom Laetiporus sulfureus. J. Biochem. 1994, 116, 519-523.

114. Wang, H.X.; Ng, T.B.; Ooi, V.E.C. Studies on purification of a lectin from fruiting bodies of the edible shiitake mushroom Lentinus edodes. Int. J. Biochem. Cell Biol. 1999, 31, 595-599.

115. Vetchinkina, E.P.; Pozdnyakova, N.N.; Nikitina, V.E. Laccase and lectin activities of intracellular proteins produced in a submerged culture of the xylotrophic basidiomycete Lentinus edodes. Curr. Microbiol. 2008, 57, 381-385. 
116. Tsivileva, O.M.; Nikitina, V.E.; Garibova, L.V.; Zav'yalova, L.A.; Ignatov, V.V. Hemagglutinating activity of the fungus Lentinus edodes (Berk.) sing "Lentinus edodes (Berk.) Pegler". Microbiology 2000, 69, 30-35.

117. Eghianruwa, Q.; Odekanyin, O.; Kuku, A. Physicochemical properties and acute toxicity studies of a lectin from the saline extract of the fruiting bodies of the shiitake mushroom, Lentinula edodes (Berk). Int. J. Biochem. Mol. Biol. 2011, 2, 309-317.

118. Sharma, S.K.; Atri, N. Comparative study on lectin activity in mycelium of wild mushroom (Lentinus) species. Middle-East J. Sci. Res. 2012, 12, 499-503.

119. Goldstein, I.J.; Winter, H.C.; Aurandt, J.; Confer, L.; Adamson, J.T.; Hakansson, K.; Remmer, H. A new alpha-galactosyl-binding protein from the mushroom Lyophyllum decastes. Arch. Biochem. Biophys. 2007, 467, 268-274.

120. Zurga, S.; Pohleven, J.; Renko, M.; Bleuler-Martinez, S.; Sosnowski, P.; Turk, D.; Kunzler, M.; Kos, J.; Sabotic, J. A novel beta-trefoil lectin from the parasol mushroom (Macrolepiota procera) is nematotoxic. FEBS J. 2014, 281, 3489-3506.

121. Loganathan, D.; Winter, H.C.; Judd, W.J.; Petryniak, J.; Goldstein, I.J. Immobilized Marasmius oreades agglutinin: Use for binding and isolation of glycoproteins containing the xenotransplantation or human type B epitopes. Glycobiology 2003, 13, 955-960.

122. Winter, H.C.; Mostafapour, K.; Goldstein, I.J. The mushroom Marasmius oreades lectin is a blood group type B agglutinin that recognizes the Galalpha 1,3Gal and Galalpha 1,3Galbeta 1,4GlcNAc porcine xenotransplantation epitopes with high affinity. J. Biol. Chem. 2002, 277, 14996-15001.

123. Rempel, B.P.; Winter, H.C.; Goldstein, I.J.; Hindsgaul, O. Characterization of the recognition of blood group B trisaccharide derivatives by the lectin from Marasmius oreades using frontal affinity chromatography-mass spectrometry. Glycoconj. J. 2002, 19, 175-180.

124. Tateno, H.; Goldstein, I.J. Partial identification of carbohydrate-binding sites of a Galalpha1, 3Galbeta1,4GlcNAc-specific lectin from the mushroom Marasmius oreades by site-directed mutagenesis. Arch. Biochem. Biophys. 2004, 427, 101-109.

125. Shimokawa, M.; Fukudome, A.; Yamashita, R.; Minami, Y.; Yagi, F.; Tateno, H.; Hirabayashi, J. Characterization and cloning of GNA-like lectin from the mushroom Marasmius oreades. Glycoconj. J. 2012, 29, 457-465.

126. Ogawa, S.; Otta, Y.; Ando, A.; Nagata, Y. A lectin from an ascomycete mushroom, Melastiza chateri: No synthesis of the lectin in mycelial isolate. Biosci. Biotechnol. Biochem. 2001, 65, 686-689.

127. Kawagishi, H.; Takagi, J.; Taira, T.; Murata, T.; Usui, T. Purification and characterization of a lectin from the mushroom Mycoleptodonoides aitchisonii. Phytochemistry 2001, 56, 53-58.

128. Matsumoto, H.; Natsume, A.; Ueda, H.; Saitoh, T.; Ogawa, H. Screening of a unique lectin from 16 cultivable mushrooms with hybrid glycoprotein and neoproteoglycan probes and purification of a novel N-acetylglucosamine-specific lectin from Oudemansiella platyphylla fruiting body. Biochim. Biophys. Acta 2001, 1526, 37-43.

129. Gold, E.R.; Balding, P. Receptor-Specific Proteins: Plant and Animal Lectins; Excerpta Medica: Amsterdam, The Netherlands, 1975. 
130. Park, J.H.; Ryu, C.S.; Kim, H.N.; Na, Y.J.; Park, H.J.; Kim, H. A sialic acid-specific lectin from the mushroom Paecilomyces Japonica that exhibits hemagglutination activity and cytotoxicity. Protein Pept. Lett. 2004, 11, 563-569.

131. Wang, H.; Ng, T.B. First report of an arabinose-specific fungal lectin. Biochem. Biophys. Res. Commun. 2005, 337, 621-625.

132. Kawagishi, H.; Wasa, T.; Murata, T.; Usui, T.; Kimura, A.; Chiba, S. Two N-acetyl-Dgalactosamine-specific lectins from Phaeolepiota aurea. Phytochemistry 1996, 41, 1013-1016.

133. Zhang, G.Q.; Sun, J.; Wang, H.X.; Ng, T.B. A novel lectin with antiproliferative activity from the medicinal mushroom Pholiota adiposa. Acta Biochim. Pol. 2009, 56, 415-421.

134. Kawagishi, H.; Abe, Y.; Nagata, T.; Kimura, A.; Chiba, S. A lectin from the mushroom Pholiota aurivella. Agric. Biol. Chem. 1991, 55, 2485-2489.

135. Kobayashi, Y.; Tateno, H.; Dohra, H.; Moriwaki, K.; Miyoshi, E.; Hirabayashi, J.; Kawagishi, H. A novel core fucose-specific lectin from the mushroom Pholiota squarrosa. J. Biol. Chem. 2012, 287, 33973-33982.

136. Suzuki, T.; Amano, Y.; Fujita, M.; Kobayashi, Y.; Dohra, H.; Hirai, H.; Murata, T.; Usui, T.; Morita, T.; Kawagishi, H. Purification, characterization, and cDNA cloning of a lectin from the mushroom Pleurocybella porrigens. Biosci. Biotechnol. Biochem. 2009, 73, 702-709.

137. Li, Y.R.; Liu, Q.H.; Wang, H.X.; Ng, T.B. A novel lectin with potent antitumor, mitogenic and HIV-1 reverse transcriptase inhibitory activities from the edible mushroom Pleurotus citrinopileatus. Biochim. Biophys. Acta 2008, 1780, 51-57.

138. Yoshida, M.; Kato, S.; Oguri, S.; Nagata, Y. Purification and properties of lectins from a mushroom, Pleurotus cornucopiae. Biosci. Biotechnol. Biochem. 1994, 58, 498-501.

139. Mahajan, R.G.; Patil, S.I.; Mohan, D.R.; Shastry, P. Pleurotus Eous mushroom lectin (PEL) with mixed carbohydrate inhibition and antiproliferative activity on tumor cell lines. J. Biochem. Mol. Biol. Biophys. 2002, 6, 341-345.

140. Kogure, T. On the specificity of mushroom Pleurotus ostreatus and Pleurotus spodoleucus extracts. Vox Sang. 1975, 29, 221-227.

141. Kobayashi, Y.; Nakamura, H.; Sekiguchi, T.; Takanami, R.; Murata, T.; Usui, T.; Kawagishi, H. Analysis of the carbohydrate binding specificity of the mushroom Pleurotus ostreatus lectin by surface plasmon resonance. Anal. Biochem. 2005, 336, 87-93.

142. Kawagishi, H.; Suzuki, H.; Watanabe, H.; Nakamura, H.; Sekiguchi, T.; Murata, T.; Usui, T.; Sugiyama, K.; Suganuma, H.; Inakuma, T.; et al. A lectin from an edible mushroom Pleurotus ostreatus as a food intake-suppressing substance. Biochim. Biophys. Acta Gen. Subj. 2000, 1474, 299-308.

143. Wang, H.; Ng, T.B. Isolation of a novel $\mathrm{N}$-acetylglucosamine-specific lectin from fresh sclerotia of the edible mushroom Pleurotus tuber-regium. Protein Expr. Purif. 2003, 29, 156-160.

144. Wang, H.; Ng, T.B.; Liu, Q. A novel lectin from the wild mushroom Polyporus adusta. Biochem. Biophys. Res. Commun. 2003, 307, 535-539.

145. Mo, H.; Winter, H.C.; Goldstein, I.J. Purification and characterization of a Neu5Acalpha26Galbeta1-4Glc/GlcNAc-specific lectin from the fruiting body of the polypore mushroom Polyporus squamosus. J. Biol. Chem. 2000, 275, 10623-10629. 
146. Zhang, B.; Palcic, M.M.; Mo, H.; Goldstein, I.J.; Hindsgaul, O. Rapid determination of the binding affinity and specificity of the mushroom Polyporus squamosus lectin using frontal affinity chromatography coupled to electrospray mass spectrometry. Glycobiology 2001, 11, $141-147$.

147. Toma, V.; Zuber, C.; Winter, H.C.; Goldstein, I.J.; Roth, J. Application of a lectin from the mushroom Polysporus squamosus for the histochemical detection of the NeuAcalpha2,6Galbeta1, 4Glc/GlcNAc sequence of N-linked oligosaccharides: A comparison with the Sambucus nigra lectin. Histochem. Cell Biol. 2001, 116, 183-193.

148. Arigi, E.; Singh, S.; Kahlili, A.H.; Winter, H.C.; Goldstein, I.J.; Levery, S.B. Characterization of neutral and acidic glycosphingolipids from the lectin-producing mushroom, Polyporus squamosus. Glycobiology 2007, 17, 754-766.

149. Ueda, H.; Kojima, K.; Saitoh, T.; Ogawa, H. Interaction of a lectin from Psathyrella velutina mushroom with $\mathrm{N}$-acetylneuraminic acid. FEBS Lett. 1999, 448, 75-80.

150. Ueda, H.; Matsumoto, H.; Takahashi, N.; Ogawa, H. Psathyrella velutina mushroom lectin exhibits high affinity toward sialoglycoproteins possessing terminal $N$-acetylneuraminic acid alpha 2,3-linked to penultimate galactose residues of trisialyl $N$-glycans. comparison with other sialic acid-specific lectins. J. Biol. Chem. 2002, 277, 24916-24925.

151. Ueda, H.; Saitoh, T.; Kojima, K.; Ogawa, H. Multi-specificity of a Psathyrella velutina mushroom lectin: Heparin/pectin binding occurs at a site different from the $\mathrm{N}$-acetylglucosamine/ $\mathrm{N}$-acetylneuraminic acid-specific site. J. Biochem. 1999, 126, 530-537.

152. Kochibe, N.; Matta, K.L. Purification and properties of an $N$-acetylglucosamine-specific lectin from Psathyrella velutina mushroom. J. Biol. Chem. 1989, 264, 173-177.

153. Hernandez, E.; Ortiz, R.; Lopez, F.; Masso, F.; Montano, L.F.; Martinage, A.; Zenteno, E. Purification and characterization of a galactose-specific lectin from Psilocybe barrerae. Phytochemistry 1993, 32, 1209-1211.

154. Zhao, S.; Zhao, Y.; Li, S.; Zhao, J.; Zhang, G.; Wang, H.; Ng, T.B. A novel lectin with highly potent antiproliferative and HIV-1 reverse transcriptase inhibitory activities from the edible wild mushroom Russula delica. Glycoconj. J. 2010, 27, 259-265.

155. Zhang, G.; Sun, J.; Wang, H.; Ng, T.B. First isolation and characterization of a novel lectin with potent antitumor activity from a Russula mushroom. Phytomedicine 2010, 17, 775-781.

156. Chumkhunthod, P.; Rodtong, S.; Lambert, S.J.; Fordham-Skelton, A.P.; Rizkallah, P.J.; Wilkinson, M.C.; Reynolds, C.D. Purification and characterization of an $\mathrm{N}$-acetyl-D-galactosaminespecific lectin from the edible mushroom Schizophyllum commune. Biochim. Biophys. Acta 2006, 1760, 326-332.

157. Han, C.H.; Liu, Q.H.; Ng, T.B.; Wang, H.X. A novel homodimeric lactose-binding lectin from the edible split gill medicinal mushroom Schizophyllum commune. Biochem. Biophys. Res. Commun. 2005, 336, 252-257.

158. Ingram, G.A.; Murray-Rochard, S.; Dougherty, A.S. Agglutinins (lectins) and lysins in extracts of cultured and noncultured fungi. Lectins Biol. Biochem. Clin. Biochem. 1988, 6, 393-400.

159. Damian, L.; Fournier, D.; Winterhalter, M.; Paquereau, L. Determination of thermodynamic parameters of Xerocomus chrysenteron lectin interactions with $\mathrm{N}$-acetylgalactosamine and Thomsen-Friedenreich antigen by isothermal titration calorimetry. BMC Biochem. 2005, 6, 11. 
160. Trigueros, V.; Lougarre, A.; Ali-Ahmed, D.; Rahbe, Y.; Guillot, J.; Chavant, L.; Fournier, D.; Paquereau, L. Xerocomus chrysenteron lectin: Identification of a new pesticidal protein. Biochim. Biophys. Acta 2003, 1621, 292-298.

161. Liu, Q.; Wang, H.; Ng, T.B. Isolation and characterization of a novel lectin from the wild mushroom Xerocomus spadiceus. Peptides 2004, 25, 7-10.

162. Liu, Q.; Wang, H.; Ng, T.B. First report of a xylose-specific lectin with potent hemagglutinating, antiproliferative and anti-mitogenic activities from a wild ascomycete mushroom. Biochim. Biophys. Acta Gen. Subj. 2006, 1760, 1914-1919.

163. Luangsa-ard, J.J.; Hywel-Jones, N.L.; Manoch, L.; Samson, R.A. On the relationships of Paecilomyces sect. Isarioidea species. Mycol. Res. 2005, 109, 581-589.

164. Kobert, R. Lehrbuch der Intoxikationen; Fredinand Enke: Stuttgart, Germany, 1893.

165. Van Eerde, A.; Grahn, E.M.; Winter, H.C.; Goldstein, I.J.; Krengel, U. Atomic-resolution structure of the alpha-galactosyl binding Lyophyllum decastes lectin reveals a new protein family found in both fungi and plants. Glycobiology 2014, doi:10.1093/glycob/cwu136.

166. Wimmerova, M.; Mitchell, E.; Sanchez, J.F.; Gautier, C.; Imberty, A. Crystal structure of fungal lectin: Six-bladed beta-propeller fold and novel fucose recognition mode for Aleuria aurantia lectin. J. Biol. Chem. 2003, 278, 27059-27067.

167. Ogawa, S.; Nakajima, E.; Nagao, H.; Ohtoshi, M.; Ando, A.; Nagata, Y. Synthesis of a lectin in both mycelia and fruit bodies of the ascomycete mushroom Aleuria aurantia. Biosci. Biotechnol. Biochem. 1998, 62, 915-918.

168. Fujihashi, M.; Peapus, D.H.; Kamiya, N.; Nagata, Y.; Miki, K. Crystal Structure of Fucose-Specific Lectin from aleuria aurantia binding ligands at three of its five sugar recognition sites. Biochemistry 2003, 42, 11093-11099.

169. Cioci, G.; Mitchell, E.P.; Chazalet, V.; Debray, H.; Oscarson, S.; Lahmann, M.; Gautier, C.; Breton, C.; Perez, S.; Imberty, A. $\beta$-Propeller crystal structure of Psathyrella velutina lectin: An integrin-like fungal protein interacting with monosaccharides and calcium. J. Mol. Biol. 2006, 357, 1575-1591.

170. Kochibe, N. Manufacture of Novel Lectin with Psathyrella velutina. Japan Patent, 88-151136, 1989.

171. Feng, L.; Sun, H.; Zhang, Y.; Li, D.-F.; Wang, D.-C. Structural insights into the recognition mechanism between an antitumor galectin AAL and the Thomsen-Friedenreich antigen. FASEB J. 2010, 24, 3861-3868.

172. Yang, N.; Li, D.-F.; Feng, L.; Xiang, Y.; Liu, W.; Sun, H.; Wang, D.-C. Structural basis for the tumor cell apoptosis-inducing activity of an antitumor lectin from the edible mushroom Agrocybe aegerita. J. Mol. Biol. 2009, 387, 694-705.

173. Ban, M.; Yoon, H.J.; Demirkan, E.; Utsumi, S.; Mikami, B.; Yagi, F. Structural basis of a fungal galectin from Agrocybe cylindracea for recognizing sialoconjugate. J. Mol. Biol. 2005, 351, 695-706.

174. Kuwabara, N.; Hu, D.; Tateno, H.; Makyio, H.; Hirabayashi, J.; Kato, R. Conformational change of a unique sequence in a fungal galectin from Agrocybe cylindracea controls glycan ligand-binding specificity. FEBS Lett. 2013, 587, 3620-3625.

175. Walser, P.J.; Haebel, P.W.; Kunzler, M.; Sargent, D.; Kues, U.; Aebi, M.; Ban, N. Structure and functional analysis of the fungal galectin CGL2. Structure 2004, 12, 689-702. 
176. Butschi, A.; Titz, A.; Walti, M.A.; Olieric, V.; Paschinger, K.; Nobauer, K.; Guo, X.; Seeberger, P.H.; Wilson, I.B.; Aebi, M.; et al. Caenorhabditis elegans $N$-glycan core beta-galactoside confers sensitivity towards nematotoxic fungal galectin CGL2. PLoS Pathog. 2010, 6, e1000717.

177. Bovi, M.; Carrizo, M.E.; Capaldi, S.; Perduca, M.; Chiarelli, L.R.; Galliano, M.; Monaco, H.L. Structure of a lectin with antitumoral properties in king bolete (Boletus edulis) mushrooms. Glycobiology 2011, 21, 1000-1009.

178. Grahn, E.; Askarieh, G.; Holmner, A.; Tateno, H.; Winter, H.C.; Goldstein, I.J.; Krengel, U. Crystal structure of the Marasmius oreades mushroom lectin in complex with a xenotransplantation epitope. J. Mol. Biol. 2007, 369, 710-721.

179. Grahn, E.M.; Winter, H.C.; Tateno, H.; Goldstein, I.J.; Krengel, U. Structural characterization of a lectin from the mushroom Marasmius oreades in complex with the blood group B trisaccharide and calcium. J. Mol. Biol. 2009, 390, 457-466.

180. Kadirvelraj, R.; Grant, O.C.; Goldstein, I.J.; Winter, H.C.; Tateno, H.; Fadda, E.; Woods, R.J. Structure and binding analysis of Polyporus squamosus lectin in complex with the Neu5Ac \{alpha\}2-6Gal\{beta\}1-4GlcNAc human-type influenza receptor. Glycobiology 2011, 21, 973-984.

181. Birck, C.; Damian, L.; Marty-Detraves, C.; Lougarre, A.; Schulze-Briese, C.; Koehl, P.; Fournier, D.; Paquereau, L.; Samama, J.P. A new lectin family with structure similarity to actinoporins revealed by the crystal structure of Xerocomus chrysenteron lectin XCL. J. Mol. Biol. 2004, 344, 1409-1420.

182. Koyama, Y.; Katsuno, Y.; Miyoshi, N.; Hayakawa, S.; Mita, T.; Muto, H.; Isemura, S.; Aoyagi, Y.; Isemura, M. Apoptosis induction by lectin isolated from the mushroom boletopsis leucomelas in U937 cells. Biosci. Biotechnol. Biochem. 2002, 66, 784-789.

183. Wong, J.H.; Wang, H.; Ng, T.B. A haemagglutinin from the medicinal fungus Cordyceps militaris. Biosci. Rep. 2009, 29, 321-327.

184. Kawagishi, H.; Nomura, A.; Mizuno, T.; Kimura, A.; Chiba, S. Isolation and characterization of a lectin from Grifola frondosa fruiting bodies. Biochim. Biophys. Acta 1990, 1034, 247-252.

185. Wang, S.X.; Zhang, G.Q.; Zhao, S.; Xu, F.; Zhou, Y.; Geng, X.L.; Liu, Y.; Wang, H.X. Purification and characterization of a novel lectin with antiphytovirus activities from the wild mushroom Paxillus involutus. Protein Pept. Lett. 2012, 20, 767-774.

186. Rouf, R.; Stephens, A.S.; Spaan, L.; Arndt, N.X.; Day, C.J.; May, T.W.; Tiralongo, E.; Tiralongo, J. G(2)/M cell cycle arrest by an N-acetyl-D-glucosamine specific lectin from Psathyrella asperospora. Glycoconj. J. 2014, 31, 61-70.

187. Wang, H.X.; Liu, W.K.; Ng, T.B.; Ooi, V.E.C.; Chang, S.T. The immunomodulatory and antitumor activities of lectins from the mushroom Tricholoma mongolicum. Immunopharmacology 1996, 31, 205-211.

188. Lin, J.Y.; Chou, T.B. Isolation and characterization of a lectin from edible mushroom, Volvariella volvacea. J. Biochem. 1984, 96, 35-40.

189. Marty-Detraves, C.; Francis, F.; Baricault, L.; Fournier, D.; Paquereau, L. Inhibitory action of a new lectin from Xerocomus chrysenteron on cell-substrate adhesion. Mol. Cell. Biochem. 2004, 258, 49-55. 
190. Tsuda, M. Purification and characterization of a lectin from the mushroom, Flammulina veltipes. J. Biochem. 1979, 86, 1463-1468.

191. Wang, H.X.; Ng, T.B. Examination of lectins, polysaccharopeptide, polysaccharide, alkaloid, coumarin and trypsin inhibitors for inhibitory activity against human immunodeficiency virus reverse transcriptase and glycohydrolases. Planta Med. 2001, 67, 669-672.

192. Entlicher, G.; Jesenska, K.; Jarsova-Dejlova, J.; Jarnik, M.; Kocourek, J. Studies on lectins LXIII. Isolation and characterization of a lectin from stinkhorn mushroom Phallus impudicus. In Lectins, Biology, Biochemistry, Clinical Biochemstry; Hansen, T.C.B., Breborowicz, J., Eds.; Walter de Gruyter: Berlin, Germany, 1985; Volume 4, pp. 491-503.

193. Kobayashi, Y.; Kawagishi, H. Fungal lectins: A growing family. Methods Mol. Biol. 2014, 1200, 15-38.

194. Houser, J.; Komarek, J.; Kostlanova, N.; Cioci, G.; Varrot, A.; Kerr, S.C.; Lahmann, M.; Balloy, V.; Fahy, J.V.; Chignard, M.; et al. A soluble fucose-specific lectin from Aspergillus fumigatus conidia - Structure, specificity and possible role in fungal pathogenicity. PLOS ONE 2013, 8, e83077.

195. Kostlanova, N.; Mitchell, E.P.; Lortat-Jacob, H.; Oscarson, S.; Lahmann, M.; Gilboa-Garber, N.; Chambat, G.; Wimmerova, M.; Imberty, A. The fucose-binding lectin from Ralstonia solanacearum. A new type of beta-propeller architecture formed by oligomerization and interacting with fucoside, fucosyllactose, and plant xyloglucan. J. Biol. Chem. 2005, 280, 27839-27849.

196. Rutenber, E.; Ready, M.; Robertus, J.D. Structure and evolution of ricin B chain. Nature 1987, $326,624-626$.

197. Hazes, B. The (QxW)3 domain: A flexible lectin scaffold. Protein Sci. 1996, 5, 1490-1501.

198. Wohlschlager, T.; Butschi, A.; Zurfluh, K.; Vonesch, S.C.; auf dem Keller, U.; Gehrig, P.; Bleuler-Martinez, S.; Hengartner, M.O.; Aebi, M.; Künzler, M. Nematotoxicity of Marasmius oreades Agglutinin (MOA) depends on glycolipid binding and cysteine protease activity. J. Biol. Chem. 2011, 286, 30337-30343.

199. Olsnes, S. The history of ricin, abrin and related toxins. Toxicon 2004, 44, 361-370.

200. Eckhardt, A.E.; Goldstein, I.J. Occurrence of alpha-D-galactosyl-containing glycoproteins on Ehrlich tumor cell membranes. Biochemistry 1983, 22, 5280-5289.

201. Sharon, N.; Lis, H. Lectins: Cell-agglutinating and sugar-specific proteins. Science 1972, 177, 949-959.

202. Nel, A.E. T-cell activation through the antigen receptor. Part 1: Signaling components, signaling pathways, and signal integration at the T-cell antigen receptor synapse. J. Allergy Clin. Immunol. 2002, 109, 758-770.

203. Ho, J.C.K.; Sze, S.C.W.; Shen, W.Z.; Liu, W.K. Mitogenic activity of edible mushroom lectins. Biochim. Biophys. Acta Gen. Subj. 2004, 1671, 9-17.

204. Greene, W.; Fleisher, T.; Waldmann, T. Suppression of human T and B lymphocyte activation by Agaricus bisporus lectin. I. Suggestive evidence for a surface "suppressor" receptor in human lymphocytes. J. Immunol. 1981, 126, 580-586.

205. Chang, H.-H.; Chien, P.-J.; Tong, M.-H.; Sheu, F. Mushroom immunomodulatory proteins possess potential thermal/freezing resistance, acid/alkali tolerance and dehydration stability. Food Chem. 2007, 105, 597-605. 
206. Ngai, P.H.; Wang, H.X.; Ng, T.B. Purification and characterization of a ubiquitin-like peptide with macrophage stimulating, antiproliferative and ribonuclease activities from the mushroom Agrocybe cylindracea. Peptides 2003, 24, 639-645.

207. Sze, S.C.; Ho, J.C.; Liu, W.K. Volvariella volvacea lectin activates mouse T lymphocytes by a calcium dependent pathway. J. Cell. Biochem. 2004, 92, 1193-1202.

208. Bottcher, M.; Grosse, F. HIV-1 protease inhibits its homologous reverse transcriptase by protein-protein interaction. Nucleic Acids Res. 1997, 25, 1709-1714.

209. Wong, J.H.; Ng, T.B. Vulgarinin, a broad-spectrum antifungal peptide from haricot beans (Phaseolus vulgaris). Int. J. Biochem. Cell Biol. 2005, 37, 1626-1632.

210. Wang, H.; Ng, T.B. Luffangulin, a novel ribosome inactivating peptide from ridge gourd (Luffa acutangula) seeds. Life Sci. 2002, 70, 899-906.

211. Barrientos, L.G.; Gronenborn, A.M. The highly specific carbohydrate-binding protein cyanovirin-N: Structure, anti-HIV/Ebola activity and possibilities for therapy. Mini Rev. Med. Chem. 2005, 5, 21-31.

212. Bhattacharya, P.; Simet, I.; Basu, S. Inhibition of human neuroblastoma DNA polymerase activities by plant lectins and toxins. Proc. Natl. Acad. Sci. USA 1979, 76, 2218-2221.

213. Koharudin, L.M.; Viscomi, A.R.; Jee, J.G.; Ottonello, S.; Gronenborn, A.M. The evolutionarily conserved family of cyanovirin-N homologs: Structures and carbohydrate specificity. Structure 2008, 16, 570-584.

214. Akkouh, O.; Ng, T.B.; Singh, S.S.; Yin, C.; Dan, X.; Chan, Y.S.; Pan, W.; Cheung, R.C. Lectins with Anti-HIV activity: A review. Molecules 2015, 20, 648-668.

215. Boyd, M.R.; Gustafson, K.R.; McMahon, J.B.; Shoemaker, R.H.; O’Keefe, B.R.; Mori, T.; Gulakowski, R.J.; Wu, L.; Rivera, M.I.; Laurencot, C.M.; et al. Discovery of cyanovirin-N, a novel human immunodeficiency virus-inactivating protein that binds viral surface envelope glycoprotein gp120: Potential applications to microbicide development. Antimicrob. Agents Chemother. 1997, $41,1521-1530$.

216. Zappe, H.; Snell, M.E.; Bossard, M.J. PEGylation of cyanovirin-N, an entry inhibitor of HIV. Adv. Drug Deliv. Rev. 2008, 60, 79-87.

217. Gueugnot, J.; Guillot, J.; Damez, M.; Coulet, M. Identification and taxonomy of human and animal leishmanias by lectin-mediated agglutination. Acta Trop. 1984, 41, 135-143.

218. Aksoy, Ü.; Ahmet, Ü. Lectins and their application to parasitology. Turk. J. Infect. 2003, 17, 513-516.

219. Gao, W.; Sun, Y.; Chen, S.; Zhang, J.; Kang, J.; Wang, Y.; Wang, H.; Xia, G.; Liu, Q.; Kang, Y. Mushroom lectin enhanced immunogenicity of HBV DNA vaccine in C57BL/6 and HBsAg-transgenic mice. Vaccine 2013, 31, 2273-2280.

220. Yazawa, S.; Furukawa, K.; Kochibe, N. Isolation of fucosyl glycoproteins from human erythrocyte membranes by affinity chromatography using Aleuria aurantia lectin. J. Biochem. 1984, 96, 1737-1742.

221. Ohlson, C.; Karlsson, J.O. Glycoproteins of axonal transport: Polypeptides interacting with the lectin from Aleuria aurantia. Brain Res. 1983, 264, 99-104.

222. Yamashita, K.; Kochibe, N.; Ohkura, T.; Ueda, I.; Kobata, A. Fractionation of L-fucose-containing oligosaccharides on immobilized Aleuria aurantia lectin. J. Biol. Chem. 1985, 260, 4688-4693. 
223. Yazawa, S.; Kochibe, N.; Asao, T. A simple procedure for isolation of tumor-associated antigens by affinity chromatography using fucose-specific Aleuria aurantia lectin. Immunol. Investig. 1990, 19, 319-327.

224. Harada, H.; Kamei, M.; Tokumoto, Y.; Yui, S.; Koyama, F.; Kochibe, N.; Endo, T.; Kobata, A. Systematic fractionation of oligosaccharides of human immunoglobulin $\mathrm{G}$ by serial affinity chromatography on immobilized lectin columns. Anal. Biochem. 1987, 164, 374-381.

225. Tao, S.-C.; Li, Y.; Zhou, J.; Qian, J.; Schnaar, R.L.; Zhang, Y.; Goldstein, I.J.; Zhu, H.; Schneck, J.P. Lectin microarrays identify cell-specific and functionally significant cell surface glycan markers. Glycobiology 2008, 18, 761-769.

(C) 2015 by the authors; licensee MDPI, Basel, Switzerland. This article is an open access article distributed under the terms and conditions of the Creative Commons Attribution license (http://creativecommons.org/licenses/by/4.0/). 